\title{
Lateral Torsional Buckling Analysis of Moment Resisting Plane Frames
}

\author{
by Arash Sahraei ${ }^{(1)}$ and Magdi Mohareb ${ }^{(1)}$
}

(1) Department of Civil Engineering, University of Ottawa, Ottawa, ON, Canada K1N 6N5

\begin{abstract}
A finite element formulation is developed to predict the lateral torsional buckling resistance of plane frames with moment connections. The solution focuses on the simple characterization the elastic warping behavior of moment connections in a manner that allows them to interface seamlessly with existing beam buckling finite elements, thus providing means for realistically modelling the lateral torsional buckling of plane frames. Special attention is devoted to the joint rotation effects. The technique successfully captures the interaction between beams and columns of frames, an effect that is neglected in present design methodologies based on individual member checks. The solution is shown to provide lateral torsional buckling resistance predictions in very good agreement with shell based finite element solutions at a fraction of the modelling and computational effort. For typical frames that are laterally supported at the joints, the study suggests that present design methodologies that isolate the member from the rest of the structure provide conservative buckling resistance predictions. Conversely, for frames with no lateral restraints at some of the joints, the present solution predicts lateral torsional buckling resistances that are significantly different from those based on design standard equations, suggesting the need to account for interaction effects in such situations.
\end{abstract}

Keywords: Lateral torsional buckling, plane frames, finite element, joint element, interaction buckling

This article is to be cited as

Sahraei A, Mohareb M, (2019) Lateral torsional buckling analysis of moment resisting plane frames, Thin-Walled Structures, $134,233-254$.

The copy-edited version of this article is available at https://doi.org/10.1016/j.tws.2018.10.006 


\section{Motivation}

Present structural steel design standards (CAN/CSA S16-14 [1], ANSI/AISC 360-16 [2]) stipulate isolating the member from the surrounding structure when investigating its capacity through interaction checks. The idea of separating a member from the surrounding structure when determining its lateral torsional buckling (LTB) resistance may be traced to the work of Salvadori [3] who advocated that such an approach yields conservative estimates for the critical moment capacity of the member. The approach of separating individual members from other members of the frame neglects interactions between various elements of the structure. Existing beam buckling finite elements provide effective means of capturing interaction effects between adjacent elements when characterising the LTB capacity of the structure, but are primarily intended to collinear structures (e.g., continuous beams). When modelling typical non-collinear frames for lateral torsional buckling, the interaction between various components can be captured by conducting a shell analysis for the whole structure, an impractical option for frames consisting of multiple storeys and/or multiple bays. Relatively recent work has extended the use existing beam buckling solutions to the LTB analysis of frames, but is limited to rather simple frame configurations. Within this context, the present study aims at developing more general means of incorporating interaction effects between various adjoining non-collinear elements (beams, columns) when characterizing their LTB resistance for multi-story frames.

\section{Literature review}

An extensive body of research has been developed for the LTB analysis of collinear beams with doubly symmetric cross-sections. Classical solutions based on the Vlasov beam theory include the work of Krajcinovic [4], Barsoum and Gallagher [5] and Powel and Klingner [6]. The classical Vlasov treatment neglects shear deformation and distortional effects. More recent solutions account for shear deformation effects and include the work of Erkmen and Mohareb [7, 8], Wu and Mohareb [9, 10], Attard and Kim [11], Erkmen and Attard [12] Erkmen [13] and Sahraei and Mohareb [14]. The LTB analysis of mono-symmetric sections include the work of Kitipornchai et al. [15], Wang and Kitipornchai [15, 16], Mohri et al. [17-19], Andrade et al. [20], Zhang and Tong [21], and Sahraei et al. [22]. Distortional lateral torsional buckling investigations, whereby the web 
distorts in addition to undergoing lateral displacement and twist (Fig. 1), include the work of Johnson and Will [23], Hancock et al. [24], Bradford and Trahair [25, 26], Bradford [27-30], Bradford and Ronagh [31] who developed finite element solutions for a variety of boundary conditions and loading cases, Hassan and Mohareb [32], Pezeshky et al. [33], and Pezeshky and Mohareb [34].

While the above studies and others have focused on the LTB analysis of collinear members, relatively fewer studies investigated the LTB capacity of non-collinear members involving beams, columns, and connecting joints. Hartmann and Munse [35] numerically investigated the LTB of portal frames with rigid joints. Vacharajittiphan and Trahair [36] developed a finite element model to examine the effect of member span, joint angle, and stiffener arrangement on the warping restraint provided by joints. Morrel [37] experimentally examined the effects of the common joint details including one, two, or three pairs of stiffeners on the torsional behavior of $\Gamma$-shaped frames containing channel cross-sections. Ettouney and Kirby [38] and Yang and McGuire [39] expressed the warping degree of freedom in terms of other degrees of freedom at a joint and adopted a warping spring to characterize the warping boundary conditions. Sharman [40] incorporated the effect of warping into the standard stiffness matrix of open section thin-walled beams by assuming the twist rate of a member to be equal to that of the adjacent member at the intersection joint in the $\Gamma$-shaped frames. Krenk and Damkidle [41] characterized the warping and distortion of connections of beams with I-shaped cross-sections. Four connections types were investigated: (1) unstiffened, (2) partially stiffened with a single diagonal stiffener, (3) a pair of perpendicular stiffeners, and (4) fully stiffened with three pairs of stiffeners. Pi and Trahair [42] investigated the effect of end-supports on the distortional lateral buckling and warping resistance of I-beams. Masarira [43] investigated the effect of joint details with various stiffener arrangements on the lateral torsional buckling of plane frames. Mohareb and Dabbas [44] and Zinoviev and Mohareb [45] showed that design procedures in standards, which neglect member interaction, are unable to predict the buckling strength of plane frames with no lateral restraints at the joints and advocated the use of shell finite element analysis for such problems. Tong et al. [46] developed a warping transmission model for beam-to-column rigid joints with diagonal stiffeners. The model captures the bending and twist effect of the diagonal stiffener and the twist restraint of the diagonal stiffener. Basaglia et al. [47] simulated the transmission of warping deformation at frame joints consisting 
of non-collinear U- and I-section members. Shayan and Rasmussen [48] developed a joint model and incorporated it into buckling finite element analysis. This joint element considers the effect of partial warping transmission between members of a plane frame. Wu and Mohareb [49] developed a joint element for LTB analysis of portal and $\Gamma$-shaped frames. The joint element can be used to characterize the stiffness of moment connections at the intersection of two members at 90 degrees. The above studies provided various treatments to characterize the partial fixity conditions at beamto-column junctions for specific frame configurations and adopted them to determine the LTB capacity of the frames as a whole. In this context, the present study is aimed to provide a computationally efficient methodology to determine the elastic LTB strength for a common class of moment resisting plane frames, which accounts for the flexibility of the joints and the effect of joint rotations.

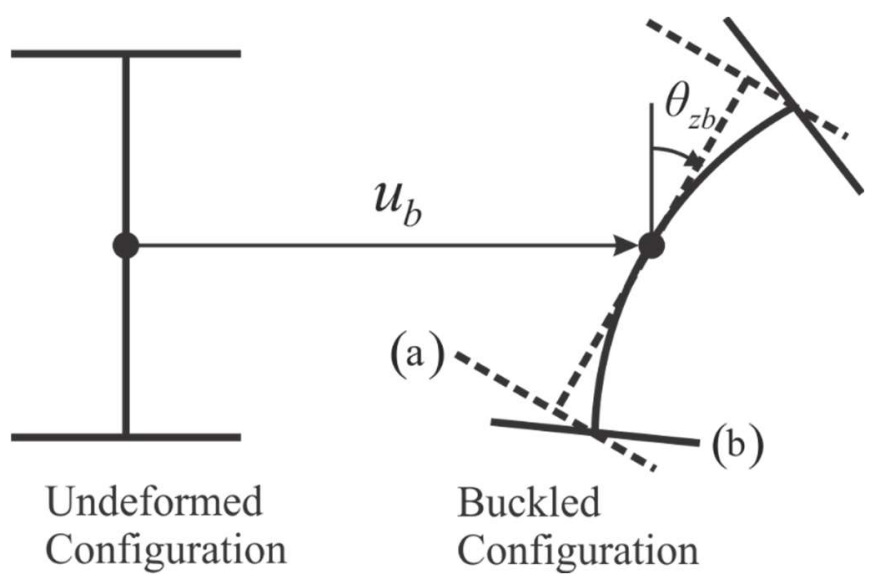

Fig. 1 Lateral torsional buckling in a classical sense (a) versus distortional lateral torsional buckling (b)

\section{Assumptions}

The following assumptions have been adopted:

1. The solution is applicable to plane frames consisting of doubly symmetric I-shaped crosssections;

2. All members are assumed to be connected at right angles through welded moment connections consisting of two pairs of horizontal and vertical stiffeners;

3. The material is assumed linearly elastic, isotropic, and obeying Hooke's law;

4. Second-order effects throughout pre-buckling deformations are assumed negligible; 
5. Throughout buckling, the joint is assumed to: (a) displace and rotate as a rigid body and (b) warp elastically along the sides of adjoining members such that they match the warping deformations of the adjoining members; and

6. All rotations under Item $5 \mathrm{a}$ are assumed moderate.

\section{Outline of the solution}

A general plane frame structure is subjected to general planar loads (Fig. 2a). The frame can be idealized as an assembly of elements (either collinear or intersecting at 90 degrees) and joint elements connecting the elements (Fig. 2b). A typical member $a b$ (Fig. 2c) has two nodes with seven degrees of freedom per node; three of them characterize the pre-buckling deformation. These are the longitudinal displacement $w$, transverse displacement $v$, and major-axis angle of rotation $\theta_{x}$, and the remaining four degrees of freedom including lateral displacement $u$, weak-axis rotation $\theta_{y}$, angle of twist $\theta_{z}$, and warping deformation $\psi$ characterize the buckling deformation (Fig. 2c). Throughout buckling, a generic joint such as $4-j k$ undergoes rigid body lateral displacement $u_{0}$ and rotations $\theta_{y 0}, \theta_{z 0}$ as shown in Fig. $2 \mathrm{~d}$. The warping stiffness of the joint is to be characterized by an independent elastic analysis based on a shell model (Fig. 2e). The degrees of freedom of the shell model of the joint are then to be condensed into four warping degrees of freedom (Fig. 2f) associated with each of the four faces of the joint. A block diagram of the steps of the proposed solution is provided in Fig. 2g. Module I loops on the straight elements to calculate their elastic and geometric stiffness matrices and send the contributions to the structure stiffness matrix. Several beam buckling elements can be adopted such as (Krajcinovic [4], Barsoum and Gallagher [5], Powel and Klingner [6], Erkmen and Mohareb [7], Wu and Mohareb [10], Sahraei and Mohareb [14], etc.). Unlike co-linear structures, where joint rotation effects are negligible [4$7,10]$, the present study incorporates joint rotation effects which will be shown to be essential in characterizing the LTB buckling resistance of laterally unsupported frames with free ends (Examples 1-4). Such joint rotation effects will be presented in Section 5.5. Module II adds the joint contributions. The kinematic constraints related to rigid body motion are to be incorporated in Module II.1 in Fig. 2g and the underlying formulation is provided under Section 5.2. For each joint, an independent shell analysis is conducted to characterize the relation between warping 
degrees of freedom applied at each of its faces (Fig. 2e) and the corresponding bimoments and incorporated in Module II.3 in Fig. 2g. The underlying formulations are provided under Section 5.3. 


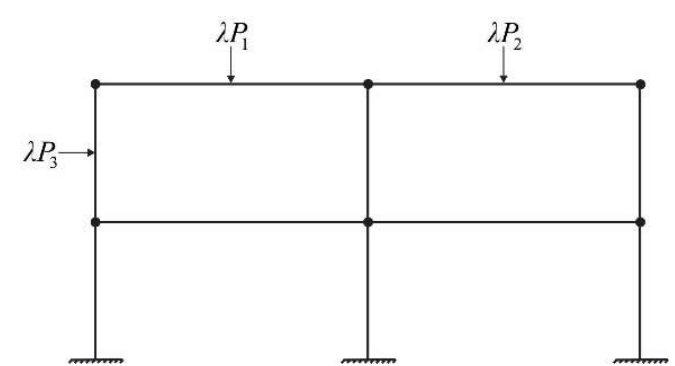

(a)

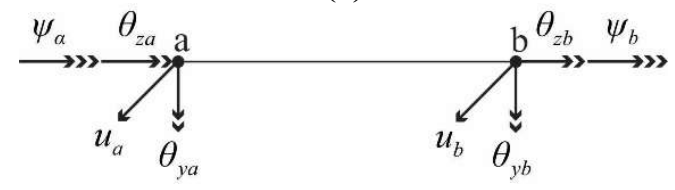

(c)

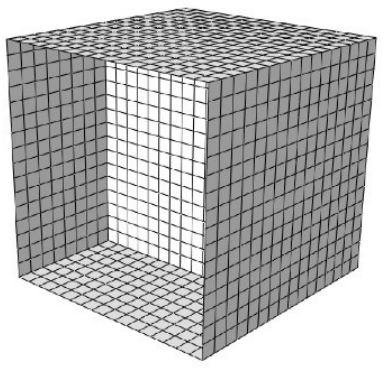

(e)

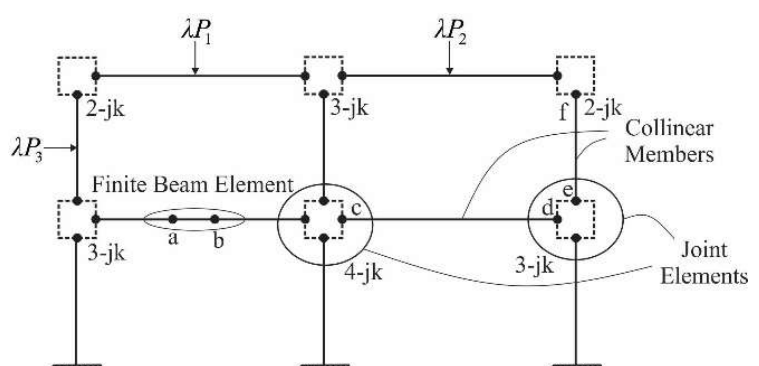

(b)

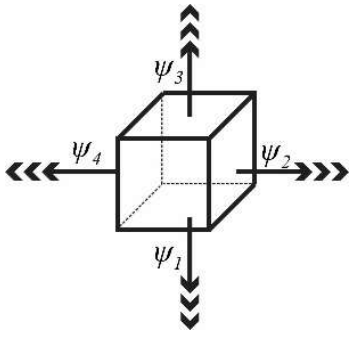

(f)

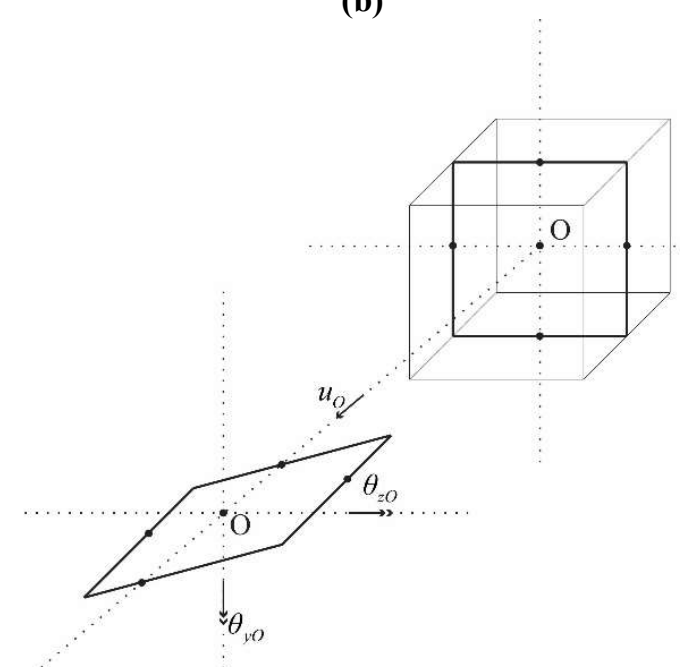

(d)

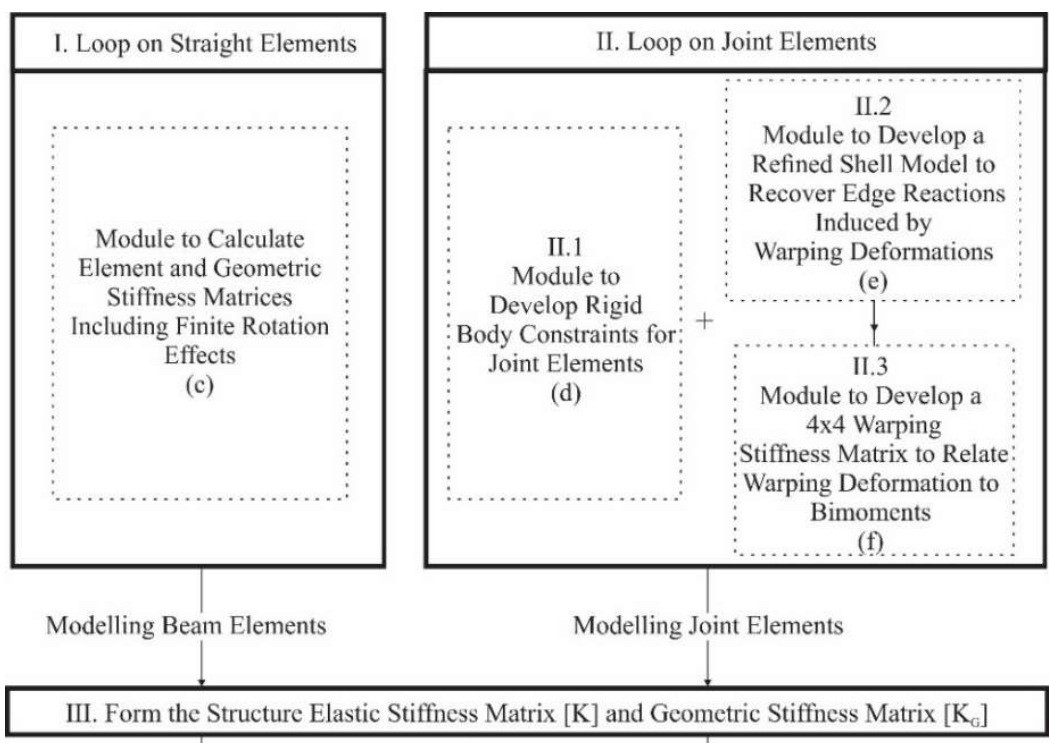

IV. Solving Buckling Eigenvalue Analysis $\left([K]+\lambda\left[K_{G}\right]\right)\left\{u_{b}\right\}=\{0\}$

(g)

Fig. 2 (a) Conventional representation of a frame, (b) Model proposed in present research, (c) DOFs of a beam finite element, (d) Rigid body displacements and rotations for joint, (e) Shell joint model, (f) Warping 


\section{deformations for joint element and (g) Block diagrams showing various components of the analysis to} conduct LTB analysis for frame

\section{Variational formulation}

The in-plane loads acting on the frame are assumed to increase until the point of onset of bucking is attained. When a frame attains this point, it reaches a state neutral stability after which it becomes prone to buckle in a lateral torsional mode (out of its plane) and the variation of the second variation of the total potential energy $\pi$ has to vanish [50] i.e.,

$\delta\left(\frac{1}{2} \delta^{2} \pi\right)=\delta\left[\frac{1}{2} \delta^{2}\left(\pi_{m}+\pi_{c}+\pi_{j}\right)\right]=0$

where $\pi_{m}=$ total potential energy for all collinear member elements undergoing elastic buckling deformations, $\pi_{c}$ is the term resulting from the Lagrange multipliers to enforce the kinematic constraints describing the rigid body motion (postulated under Assumption 5a), which relate the displacements of node 1 to those of nodes 2-4 within each joint element, and $\pi_{j}=$ total potential energy of the joint elements undergoing elastic buckling warping deformation (as per Assumption $5 \mathrm{~b}$ and Fig. 3). Symbol $\delta$ denotes the variation of the argument functionals with respect to the buckling displacements. The following three sections formulate the expressions for $\pi_{m}, \pi_{c}$ and $\pi_{j}$ respectively.

\subsection{Stiffness matrices for members}

The second variation of the total potential energy of the members, $\delta^{2} \pi_{m}$ takes the form

$$
\begin{aligned}
\frac{1}{2} \delta^{2} \pi_{m} & =\sum_{i=1}^{n} \frac{1}{2}\left\langle\delta\left({ }^{e i} U_{n l}\right)\right\rangle^{T}\left\{\left[{ }^{e i} K\right]-\lambda\left[{ }^{e i} K_{G}\right]\right\}\left\{\delta\left({ }^{e i} U_{n l}\right)\right\} \\
& =\sum_{i=1}^{n} \frac{1}{2}\left\langle\delta\left({ }^{e i} U_{n l}\right)\right\rangle^{T}\left[T\left(\varphi_{e i}\right)\right]^{T}\left\{\left[{ }^{e i} K\right]-\lambda\left[{ }^{e i} K_{G}\right]\right\}\left[T\left(\varphi_{e i}\right)\right]\left\{\delta\left({ }^{e i} U_{n l}\right)\right\}
\end{aligned}
$$

in which, $\left\langle\delta\left({ }^{e i} U_{n l}\right)\right\rangle^{T}=\left\langle{ }_{1}^{e i} u_{b l} \quad{ }_{1}^{e i} \theta_{y b l} \quad{ }_{1}^{e i} \theta_{z b l} \quad{ }_{1}^{e i} \psi_{b l} \mid{ }_{2}^{e i} u_{b l} \quad{ }_{2}^{e i} \theta_{y b l} \quad{ }_{2}^{e i} \theta_{z b l} \quad{ }_{2}^{e i} \psi_{b l}\right\rangle$ denotes the buckling nodal displacement of the member and are expressed in terms of the local coordinates. As a notation convention, symbols $e$ in the left superscripts denote that the generalized nodal displacements pertaining to an element (as opposed to a joint), and $i=1, \ldots, n$ denotes the element 
number. In the notation of the nodal displacements, the left subscript takes the values 1 or 2 to denote the first or second nodes of the element, respectively. Identifier $l$ in the right subscript indicates that nodal displacements $\left\{U_{n l}\right\}$ are defined in local coordinates, while identifier $g$ denotes that nodal displacements $\left\{U_{n g}\right\}$ are defined in global coordinates. The entries of the stiffness matrices $\left[{ }^{e i} K\right]$ and $\left[{ }^{e i} K_{G}\right]_{C}$ can be adopted from any of past solutions (e.g., [5], [7], [10] and [14]). In the present study, the classical solution in [5] and the shear deformable SM-M element in [14] are adopted. In Eq. (2), the nodal displacements $\left\{{ }^{e i} U_{n l}\right\}$ in local coordinates are related to the nodal displacements $\left\{{ }^{e i} U_{n g}\right\}$ in global coordinates through the transformation $\left\{{ }^{e i} U_{n l}\right\}_{8 \times 1}=\left[T\left(\varphi_{e i}\right)\right]_{8 \times 8}\left\{{ }^{e i} U_{n g}\right\}_{8 \times 1}$

where

$$
\left[T\left(\varphi_{e i}\right)\right]_{8 \times 8}=\left[\begin{array}{cccccccc}
1 & 0 & 0 & 0 & 0 & 0 & 0 & 0 \\
0 & \cos \varphi_{e i} & \sin \varphi_{e i} & 0 & 0 & 0 & 0 & 0 \\
0 & -\sin \varphi_{e i} & \cos \varphi_{e i} & 0 & 0 & 0 & 0 & 0 \\
0 & 0 & 0 & 1 & 0 & 0 & 0 & 0 \\
0 & 0 & 0 & 0 & 1 & 0 & 0 & 0 \\
0 & 0 & 0 & 0 & 0 & \cos \varphi_{e i} & \sin \varphi_{e i} & 0 \\
0 & 0 & 0 & 0 & 0 & -\sin \varphi_{e i} & \cos \varphi_{e i} & 0 \\
0 & 0 & 0 & 0 & 0 & 0 & 0 & 1
\end{array}\right]_{8 \times 8}
$$

and angle $\varphi_{e i}=$ counter clockwise rotation angle from the global $z_{g}$ direction to the local $z_{e i}$ direction for member $i$. For frames exclusively consisting of horizontal and vertical members, $\varphi_{e i}$ takes one of the values $0, \pi / 2, \pi, 3 \pi / 2$. As a notation convention, total field variables (i.e., those describing the motion from the initial state (Configuration 1 in Fig. 3) to the buckled state (Configuration 4) will be denoted by superscript *, variables with subscripts $b$ denote buckling variables (i.e., those describing the motion from the onset of buckling (Configuration 3) to the buckled state in Configuration 4), while those with a superscript $p$ denote pre-buckling displacements (i.e., those describing the motion from the initial state (Configuration 1) to the prebuckled state (Configuration 2). Variables without subscripts or a superscript * are generic and applicable to pre-buckling, buckling, or total field variables. 


\subsection{Kinematic constraints at the joints}

When a laterally unsupported plane frame undergoes in-plane major-axis bending, a generic joint $k$ is assumed to undergo rigid body displacements within the plane of the frame from the initial state (Configuration 1 in Fig. 3) to an equilibrium state (Configuration 2). This pre-buckling movement is characterized by two orthogonal displacements ${ }_{2}^{j k} v_{p l}$ and ${ }_{2}^{j k} w_{p l}$ at an arbitrary point (e.g., Node 2) and a rotation angle ${ }_{2}^{j k} \theta_{x p l}$. Subscript $l$ denotes displacements that are defined in the local coordinates of the joint. In the left superscript, $j$ denotes that the generalized displacement is related to a joint (as opposed to a member), and $k$ denotes the joint number. The left subscript takes the values $1, \ldots, 4$ to denote the first through fourth nodes of the joint element, respectively. When the loads are magnified by factor $\lambda$, the structure is assumed to reach the state of onset of buckling (Configuration 3), and the associated displacements are assumed to become $\lambda\left({ }_{2}^{j k} v_{p l}\right)$, $\lambda\left({ }_{2}^{j k} w_{p l}\right)$ and $\lambda\left({ }_{2}^{j k} \theta_{x p l}\right)$. This assumption neglects pre-buckling second order effects (in line with Assumption 4). 


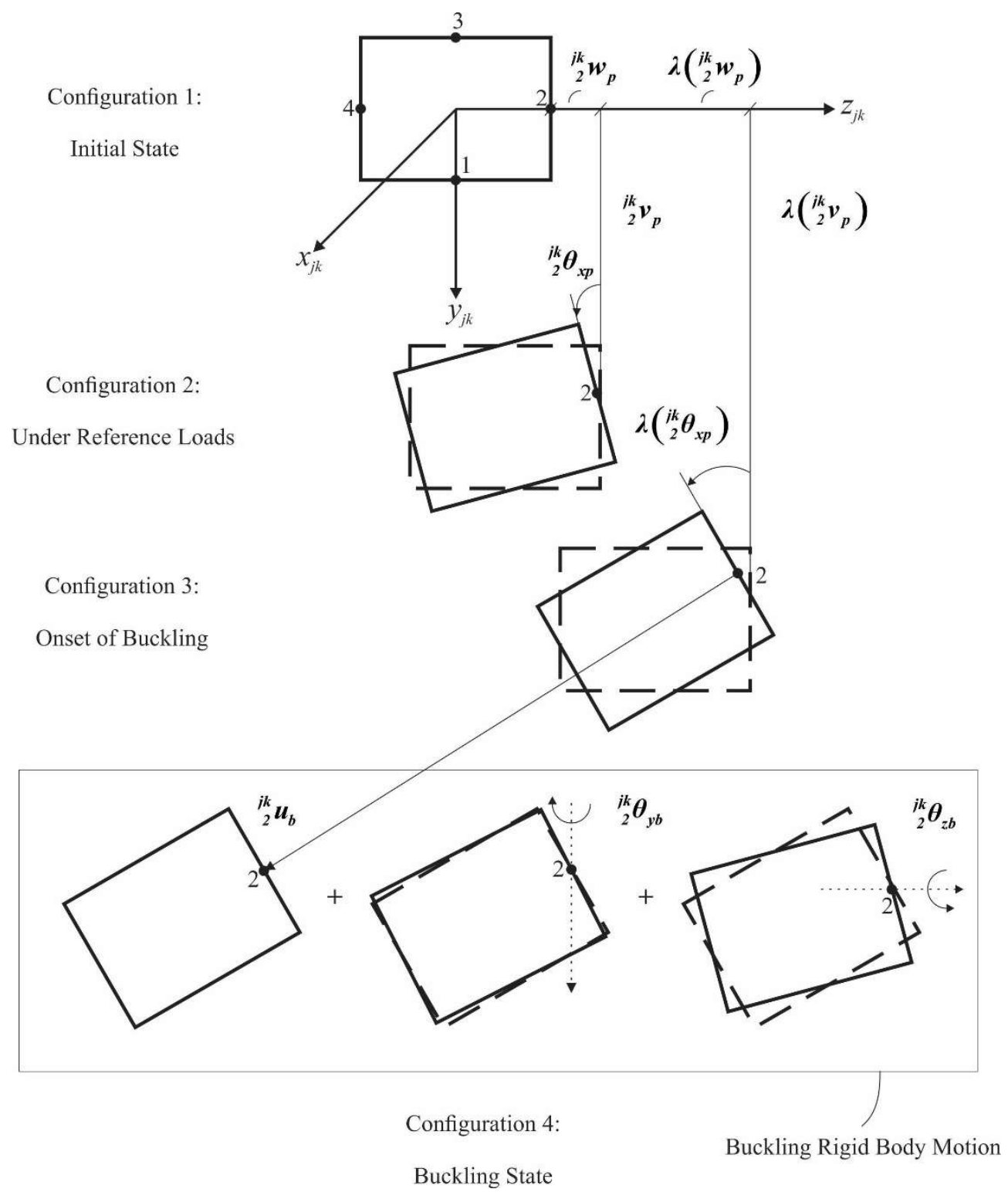

Fig. 3 Rigid body motion of a joint from initial state to buckled configuration

When the frame buckles laterally, the joint undergoes additional rigid body motion consisting of out of plane displacement ${ }_{2}^{j k} u_{b l}$ and two rotations ${ }_{2}^{j k} \theta_{y b l}$ and ${ }_{2}^{j k} \theta_{z b l}$ (Fig. 4). In addition, the joint undergoes elastic warping deformations ${ }_{1}^{j k} \psi_{b l}$ through ${ }_{4}^{j k} \psi_{b l}$ at Faces 1 through 4 which are connected to Elements 1 through 4, respectively. Warping deformation ${ }_{1}^{j k} \psi_{b l}$ induces angles of rotation ${ }_{1}^{j k} \theta_{\psi b l}$ at edge $5^{\prime}-5^{\prime \prime}$ and $-{ }_{1}^{j k} \theta_{\psi b l}$ at edge $8^{\prime}-8^{\prime \prime}$. Also, warping deformation ${ }_{2}^{j k} \psi_{b l}$ induces angles of rotation ${ }_{2}^{j k} \theta_{\psi b l}$ and $-{ }_{2}^{j k} \theta_{\psi b l}$ at edges $5^{\prime}-5^{\prime \prime}$ and $6^{\prime}-6^{\prime \prime}$. Similarly, warping deformation 
${ }_{3}^{j k} \psi_{b l}$ induces angles rotation ${ }_{3}^{j k} \theta_{\psi b l}$ and $-{ }_{3}^{j k} \theta_{\psi b l}$ at edges $6^{\prime}-6^{\prime \prime}$ and $7^{\prime}-7^{\prime \prime}$, and warping deformation ${ }_{4}^{j k} \psi_{b l}$ induces an angles of rotation ${ }_{4}^{j k} \theta_{\psi b l}$ and $-{ }_{4}^{j k} \theta_{\psi b l}$ at $8^{\prime}-8^{\prime \prime}$ and $7^{\prime}-7^{\prime \prime}$. The joint element consists of four nodes, each having three pre-buckling and four buckling generalized displacements. In local coordinates, the pre-buckling nodal displacement vector is $\left\{{ }^{j k} U_{p l}\right\}=\left\langle\begin{array}{lll|lllllllllll}{ }_{1}^{j k} v_{p l} & { }_{1}^{j k} w_{p l} & { }_{1}^{j k} \theta_{x p l} & \mid{ }_{2}^{j k} v_{p l} & { }_{2}^{j k} w_{p l} & { }_{2}^{j k} \theta_{x p l} & { }_{3}^{j k} v_{p l} & { }_{3}^{j k} w_{p l} & { }_{3}^{j k} \theta_{x p l} & \mid{ }_{4}^{j k} v_{p l} & { }_{4}^{j k} w_{p l} & { }_{4}^{j k} \theta_{x p l}\end{array}\right\rangle^{T}$ and the buckling displacement vector is

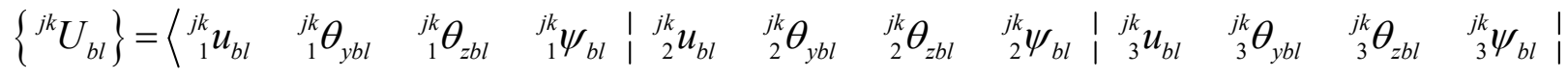

$$
\begin{aligned}
& \left.{ }_{4}^{j k} u_{b l} \quad{ }_{4}^{j k} \theta_{y b l} \quad{ }_{4}^{j k} \theta_{z b l} \quad{ }_{4}^{j k} \psi_{b l}\right\rangle^{T}
\end{aligned}
$$

Each node of the joint element is intended to interface with that of an adjoining beam element. For example, the first node (Fig. 5) connects to the end of element $e_{1}$ through global node $N_{1}$ which has global nodal displacements ${ }^{N_{1}} u_{b g},{ }^{N_{1}} \theta_{y b g},{ }^{N_{1}} \theta_{z b g}$ and ${ }^{N_{1}} \psi_{b g}$. Likewise, the second node connects to the end of element $e_{2}$ through global node $N_{2}$ with nodal displacements ${ }^{N_{2}} u_{b g},{ }^{N_{2}} \theta_{y b g},{ }^{N_{2}} \theta_{z b g}$ and ${ }^{N_{2}} \psi_{b g}$.

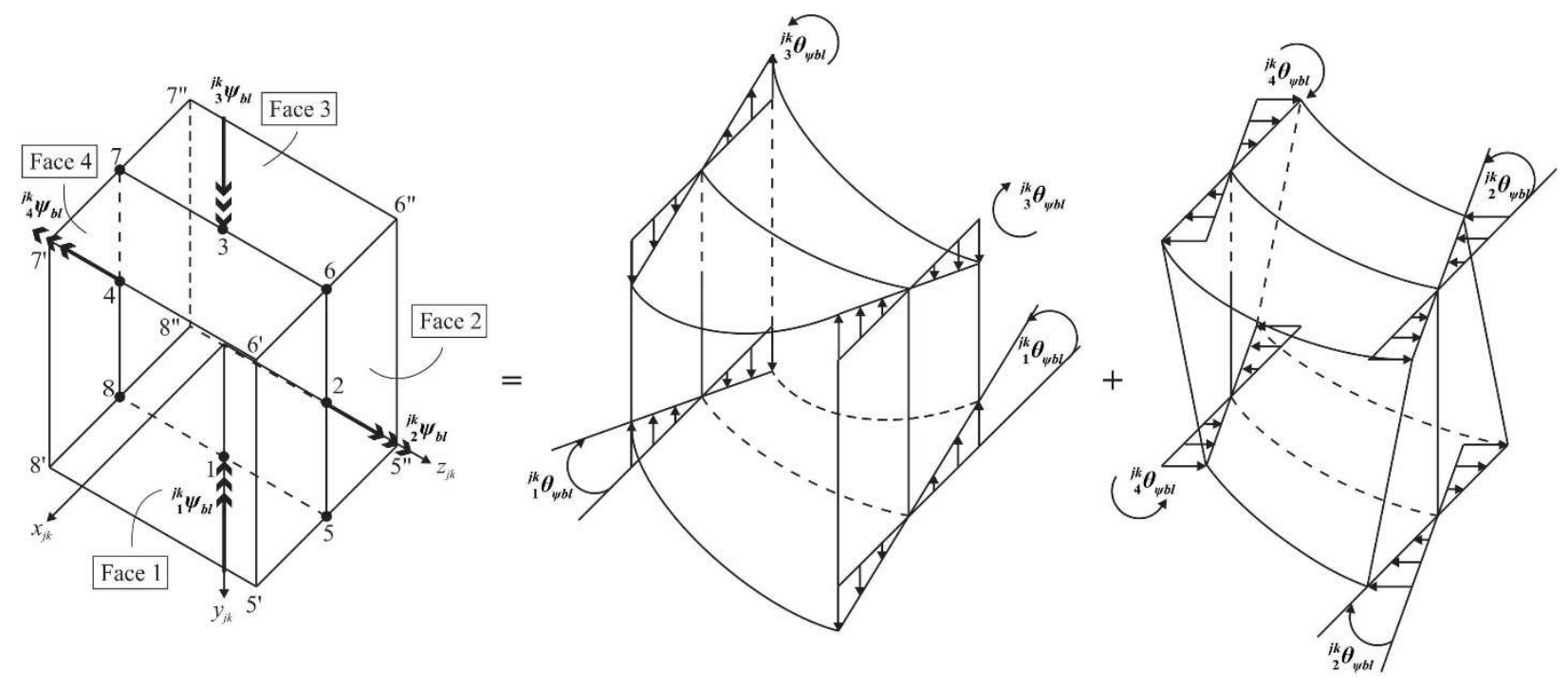

Fig. 4 Warping deformations of joint $k$ and sign conventions

The plane frame is assumed to consist of $i=1,2, \ldots, n$ members, $k=1,2, \ldots, m$ joints, and $o$ nodes. As shown in Fig. 5, each member element has its own element local coordinate system 
$\left(x_{e i}, y_{e i}, z_{e i}\right)$ Also, each joint element $k$ has its own local coordinate system $\left(x_{j k}, y_{j k}, z_{j k}\right)$. Coordinate system $\left(x_{e i}, y_{e i}, z_{e i}\right)$ defines the positive directions of the nodal displacements within member element $i$, while coordinate system $\left(x_{j k}, y_{j k}, z_{j k}\right)$ defines the positive directions of the nodal displacements within joint element $k$. Finally, the nodal degrees of freedom at nodes $1,2, \ldots, o$ are oriented along global coordinate $\operatorname{system}\left(x_{g}, y_{g}, z_{g}\right)$. 

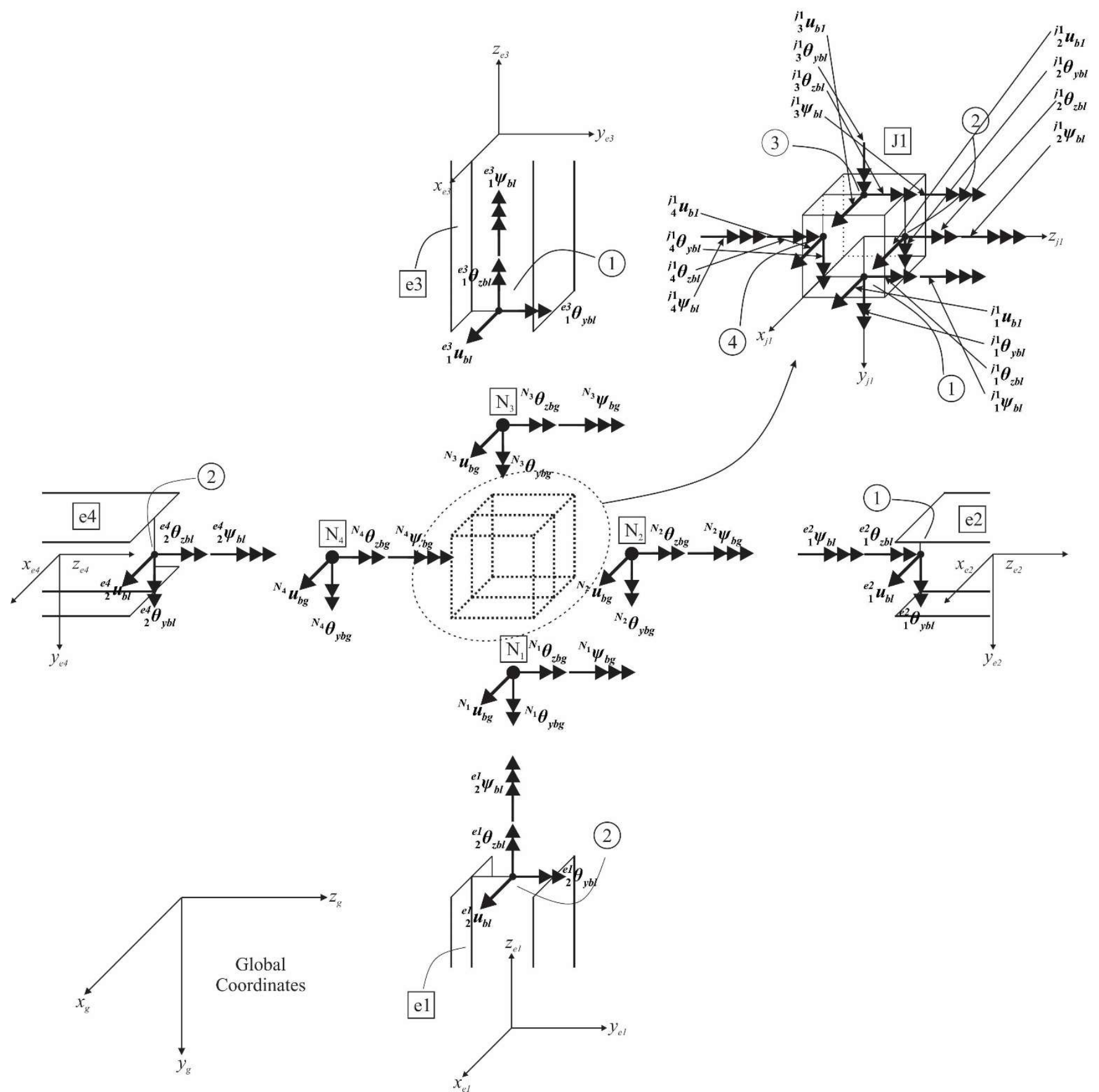

Fig. 5 Connectivity of joint and members (displacements of Global nodes N1-N4 are shown in global directions, displacements of joint $\mathrm{J} 1$ and elements e1-e4 are shown in local directions)

The three pre-buckling degrees of freedom (DOFs) for each of the nodes $i=2,3,4$ (Fig. 3) can be related those of node 1, considered as the master node. Nine pre-buckling rigid body constraints can be expressed in the form 


$$
\begin{aligned}
& v_{p i}=v_{p 1}+\left(z_{i}-z_{1}\right) \theta_{x p i 1} \\
& w_{p i}=w_{p 1}-\left(y_{i}-y_{1}\right) \theta_{x p i 1} \\
& \theta_{x p i}=\theta_{x p 1} \quad i=2,3,4
\end{aligned}
$$

Based on Fig. 6, for a given joint $k=1, \ldots, m$ with local coordinate $\operatorname{system}\left(x_{j k}, y_{j k}, z_{j k}\right)$, position vectors $\left\{x_{1}\right\}$ to $\left\{x_{8}\right\}$ in the un-deformed configuration for points 1 to 8 are given by

$$
\begin{aligned}
& \left\{x_{1}\right\}=\left\{\begin{array}{c}
0 \\
h_{2} / 2 \\
0
\end{array}\right\}, \quad\left\{x_{2}\right\}=\left\{\begin{array}{c}
0 \\
0 \\
h_{1} / 2
\end{array}\right\}, \quad\left\{x_{3}\right\}=\left\{\begin{array}{c}
0 \\
\left.-h_{2} / 2\right\} \\
0
\end{array}\right\}, \quad\left\{x_{4}\right\}=\left\{\begin{array}{c}
0 \\
0 \\
-h_{1} / 2
\end{array}\right\} \\
& \left\{x_{5}\right\}=\left\{\begin{array}{c}
0 \\
h_{2} / 2 \\
h_{1} / 2
\end{array}\right\}, \quad\left\{x_{6}\right\}=\left\{\begin{array}{c}
0 \\
-h_{2} / 2 \\
h_{1} / 2
\end{array}\right\}, \quad\left\{x_{7}\right\}=\left\{\begin{array}{c}
0 \\
-h_{2} / 2 \\
-h_{1} / 2
\end{array}\right\}, \quad\left\{x_{8}\right\}=\left\{\begin{array}{c}
0 \\
h_{2} / 2 \\
-h_{1} / 2
\end{array}\right\}
\end{aligned}
$$

from which position vector $\left\langle x_{21}\right\rangle^{T}$ for point 2 relative to point 1 is given by

$$
\left\langle x_{21}\right\rangle^{T}=\left\langle x_{2}\right\rangle^{T}-\left\langle x_{1}\right\rangle^{T}=\left\langle\begin{array}{lll}
0 & -h_{2} / 2 & \left.h_{1} / 2\right\rangle
\end{array}\right.
$$

Also, position vectors $\left\langle x_{31}\right\rangle^{T}$ and $\left\langle x_{41}\right\rangle^{T}$ for points 3 and 4 relative to point 1 are

$$
\begin{aligned}
& \left\langle x_{31}\right\rangle^{T}=\left\langle x_{3}\right\rangle^{T}-\left\langle x_{1}\right\rangle^{T}=\left\langle\begin{array}{lll}
0 & -h_{2} & 0
\end{array}\right) \\
& \left\langle x_{41}\right\rangle^{T}=\left\langle x_{4}\right\rangle^{T}-\left\langle x_{1}\right\rangle^{T}=\left\langle\begin{array}{lll}
0 & -h_{2} / 2 & -h_{1} / 2
\end{array}\right.
\end{aligned}
$$




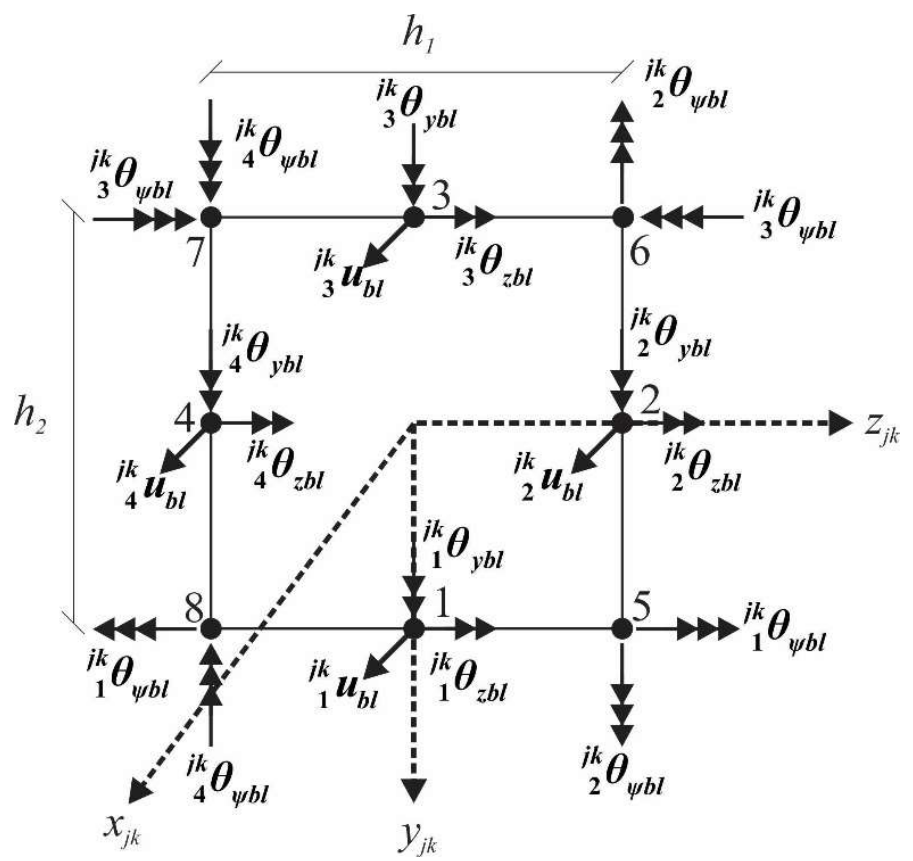

Fig. 6 Buckling degrees of freedom for a joint element in local coordinates (displacements are shown as single-headed arrows, rotations as double-headed arrows, and rotations induced by warping triple-headed arrows)

Rigid body constraints are such that buckling rotations at nodes 1 and $i=2,3,4$ must be identical in line with assumption (5a), i.e.,

$$
\begin{array}{ll}
\left(-{ }_{i}^{j k} \theta_{y b}+{ }_{1}^{j k} \theta_{y b}\right)=0 & i=2,3,4 \\
\left(-{ }_{i}^{j k} \theta_{z b}+{ }_{1}^{j k} \theta_{z b}\right)=0 & i=2,3,4
\end{array}
$$

The lateral displacement ${ }_{i}^{j k} u_{b}$ of nodes $i=2,3,4$ is expressed in the terms of other nodal displacements at node $1\left({ }_{1}^{j k} u_{b},{ }_{1}^{j k} \theta_{y b},{ }_{1}^{j k} \theta_{z b}\right)$ and warping displacements ${ }_{1}^{j k} \psi_{b}$ and ${ }_{i}^{j k} \psi_{b}$. First, displacement vector $\left\langle u_{i}\right\rangle^{T}=\left\langle{ }_{i}^{j k} u_{b}^{*} \quad{ }_{i}^{j k} v_{p}^{*}{ }_{i}^{j k} w_{p}^{*}\right\rangle^{T}$ at node $i$ due to rigid body displacements $\left\langle u_{1}\right\rangle^{T}=\left\langle{ }_{1}^{j k} u_{b}^{*}{ }_{1}^{j k} v_{p}^{*}{ }_{1}^{j k} w_{p}^{*}\right\rangle^{T}$ and rotations at node 1 is obtained through

$$
\left\{u_{i}\right\}_{3 \times 1}=\left\{u_{1}\right\}_{3 \times 1}+[R]_{3 \times 3}\left\{x_{i 1}\right\}_{3 \times 1}
$$

where $[R]$ is the moderate rotation matrix [49] given as 


$$
[R]_{3 \times 3}=\left[\begin{array}{ccc}
-\frac{1}{2}\left[\left({ }_{1}^{j k} \theta_{y b}\right)^{2}+\left({ }_{1}^{j k} \theta_{z b}\right)^{2}\right] & -{ }_{1}^{j k} \theta_{z b}+\frac{1}{2} \lambda\left({ }_{1}^{j k} \theta_{x p}\right){ }_{1}^{j k} \theta_{y b} & { }_{1}^{j k} \theta_{y b}+\frac{1}{2} \lambda\left({ }_{1}^{j k} \theta_{x p}\right){ }_{1}^{j k} \theta_{z b} \\
{ }^{j k} \theta_{z b}+\frac{1}{2} \lambda\left({ }_{1}^{j k} \theta_{x p}\right){ }_{1}^{j k} \theta_{y b} & -\frac{1}{2}\left[\lambda^{2}\left({ }_{1}^{j k} \theta_{x p}\right)^{2}+\left({ }_{1}^{j k} \theta_{z b}\right)^{2}\right] & -\lambda\left({ }_{1}^{j k} \theta_{x p}\right)+\frac{1}{2}{ }_{1}^{j k} \theta_{y b}{ }_{1}^{j k} \theta_{z b} \\
-{ }_{1}^{j k} \theta_{y b}+\frac{1}{2} \lambda\left({ }_{1}^{j k} \theta_{x p}\right){ }_{1}^{j k} \theta_{z b} & \lambda\left({ }_{1}^{j k} \theta_{x p}\right)+\frac{1}{2}{ }_{1}^{j k} \theta_{y b}{ }_{1}^{j k} \theta_{z b} & -\frac{1}{2}\left[\lambda^{2}\left({ }_{1}^{j k} \theta_{x p}\right)^{2}+\left({ }_{1}^{j k} \theta_{y b}\right)^{2}\right.
\end{array}\right]
$$

Lateral displacements of nodes 2-4 $\left({ }_{i}^{j k} u_{b}^{*}, \quad i=2,3,4\right)$ are related to the lateral displacement of node $1{ }_{1}^{j k} u_{b}^{*}$ by expanding the first row resulting from Eqs. (11a-c), yielding

$$
\begin{aligned}
& { }_{2}^{j k} u_{b}^{*}={ }_{1}^{j k} u_{b}^{*}+\frac{h_{2}}{2}\left({ }_{1}^{j k} \theta_{z b}\right)+\frac{h_{1}}{2}\left({ }_{1}^{j k} \theta_{y b}\right) \\
& { }_{3}^{j k} u_{b}^{*}={ }_{1}^{j k} u_{b}^{*}+h_{2}\left({ }_{1}^{j k} \theta_{z b}\right) \\
& { }_{4}^{j k} u_{b}^{*}={ }_{1}^{j k} u_{b}^{*}+\frac{h_{2}}{2}\left({ }_{1}^{j k} \theta_{z b}\right)-\frac{h_{1}}{2}\left({ }_{1}^{j k} \theta_{y b}\right)
\end{aligned}
$$

in which, one recalls that superscripts * denotes the total field variables of a node (i.e., going from configuration 1 to 4 as shown in Fig. 3. It is noted that the pre-buckling rotation term is neglected in the context of linearized buckling solutions $[5,9]$. Thus, terms containing $\theta_{x p}$ have been neglected in Eq. (11a-c). Besides the displacements derived in Section 5.2, warping deformation $\left({ }_{i}^{j k} \psi_{b} i=1,2,3,4\right)$ induce the following additional lateral displacements at faces 2 , 3 , and 4 (Appendix A).

$$
\begin{aligned}
& -{ }_{2}^{j k} u_{b}+{ }_{1}^{j k} u_{b}-\frac{h_{1} h_{2}}{4}\left({ }_{2}^{j k} \psi_{b}\right)+\frac{h_{1}}{2}\left({ }_{1}^{j k} \theta_{y b}\right)+\frac{h_{2}}{2}\left({ }_{1}^{j k} \theta_{z b}\right)+\frac{h_{1} h_{2}}{4}\left({ }_{1}^{j k} \psi_{b}\right) \approx 0 \\
& -{ }_{3}^{j k} u_{b}+{ }_{1}^{j k} u_{b}+h_{2}\left({ }_{1}^{j k} \theta_{z b}\right)+\frac{h_{1} h_{2}}{4}\left({ }_{1}^{j k} \psi_{b}\right)+\frac{h_{1} h_{2}}{4}\left({ }_{3}^{j k} \psi_{b}\right) \approx 0 \\
& -{ }_{4}^{j k} u_{b}+{ }_{1}^{j k} u_{b}+\frac{h_{1} h_{2}}{4}\left({ }_{4}^{j k} \psi_{b}\right)-\frac{h_{1}}{2}\left({ }_{1}^{j k} \theta_{y b}\right)+\frac{h_{2}}{2}\left({ }_{1}^{j k} \theta_{z b}\right)+\frac{h_{1} h_{2}}{4}\left({ }_{1}^{j k} \psi_{b}\right) \approx 0
\end{aligned}
$$

By grouping Eqs. (9), (10), (13a-c) and (14a-c), one obtains 12 constraint equations for the fourfaced joint. Constraints for all joints $k$ are then assembled in the matrix form

$$
[B]_{j k}\left\{{ }^{k} U_{b g}\right\}=\{0\}
$$

where the entries of $[B]_{j k}$ depend on the width $h_{1}$ and height $h_{2}$ of joint element $k$ and $\left\{{ }^{k} U_{b g}\right\}$ is the nodal buckling displacement vector of the joint $k$ in the global coordinates. The constraints in 
Eq. (15) are enforced through a Lagrange multiplier vector $\left\{L_{j}\right\}$. By summation over the joints in the frame, one obtains the resulting energy term

$$
\pi_{c}=\sum_{j=1}^{m}\left\langle L_{j}\right\rangle^{T}\left[B_{j k}\right]\left\{{ }^{k} U_{b g}\right\}
$$

which is the one sought in Eq. (1) to augment the total potential energy functional. It ensures that the functional is minimized subject to the constraints provided in Eq. (15).

\subsection{Warping stiffness matrices for joints connecting four elements}

As a member of a frame undergoes LTB, it tends to warp at its ends. This action is in part elastically restrained by the adjacent joint. This section aims at characterizing such warping restraint and devising a simplified warping stiffness matrix for the joint element. One recalls that, in addition to the rigid body motion previously characterizing the joint behaviour, the joint has four warping degrees of freedom (Fig. 4) ${ }_{1}^{j k} \psi_{b l}-{ }_{4}^{j k} \psi_{b l}$ at Faces 1-4, respectively. Such warping degrees of freedom need to be related to the corresponding bimoments ${ }_{1}^{j k} B_{b l}$ through ${ }_{4}^{j k} B_{b l}$ acting at Faces 14 through a $4 \times 4$ warping stiffness matrix. The warping-bimoment relationships of the joint are obtained from a separate shell analysis for the five-plate assembly of the joint. (Fig. 2e). To obtain the warping stiffness matrix of joint $k$ (Fig. 7), the joint is first subjected to a unit warping deformation ${ }_{1}^{j k} \psi_{b l}=1$ at Face 1. The warping ${ }_{1}^{j k} \psi_{b l}=1$ is applied by applying a rotation angle ${ }_{1}^{j k} \theta_{\psi b l}=h_{1} / 2 \times{ }_{1}^{j k} \psi_{b l}=h_{1} / 2$ about axis $z_{j k}$ at edge $5^{\prime}-5^{\prime \prime}$ and an equal but opposite angle $-{ }_{1}^{j k} \theta_{\psi b l}=-h_{1} / 2$ at edge $8^{\prime}-8^{\prime \prime}$ (Fig. 7a), while setting the warping deformations ${ }_{2}^{j k} \psi_{b l}{ }_{4}^{j k} \psi_{b l}$ to zero at Faces 2- 4 (by restraining the rotations of edges $6^{\prime}-6^{\prime \prime}$ and $7^{\prime}-7^{\prime \prime}$ in Fig. $7 \mathrm{~b}$ along the $z_{j k}$ direction and edges $5^{\prime}-5^{\prime \prime}, 6^{\prime}-6^{\prime \prime}, 7^{\prime}-7^{\prime \prime}$ and $8^{\prime}-8^{\prime \prime}$ along the $y_{j k}$ direction). The corresponding bimoment ${ }_{11}^{j k} B_{b l}$ induced at Face 1 is determined from the reactions ${ }_{1}^{j k} R_{y r r^{\prime}-5^{n}},{ }_{1}^{j k} R_{y r 8^{\prime}-8^{\prime \prime}}(r=1, \ldots, \alpha)$ (Fig. 7b) obtained from the shell finite element model, that is

$$
{ }_{11}^{j k} B_{b l}=\int_{A_{1}} \sigma_{1} \omega_{1}(s) d A \approx \sum_{r=1}^{n r}\left({ }^{j k} R_{y r 5^{\prime}-5^{n}}\right)\left(\frac{h_{1}}{2} x_{r 5^{\prime}-5^{\prime \prime}}\right)+\sum_{r=1}^{n r}\left({ }^{j k} R_{y r r^{\prime}-8^{n}}\right)\left(\frac{-h_{1}}{2} x_{r 8^{\prime}-8^{\prime \prime}}\right)
$$



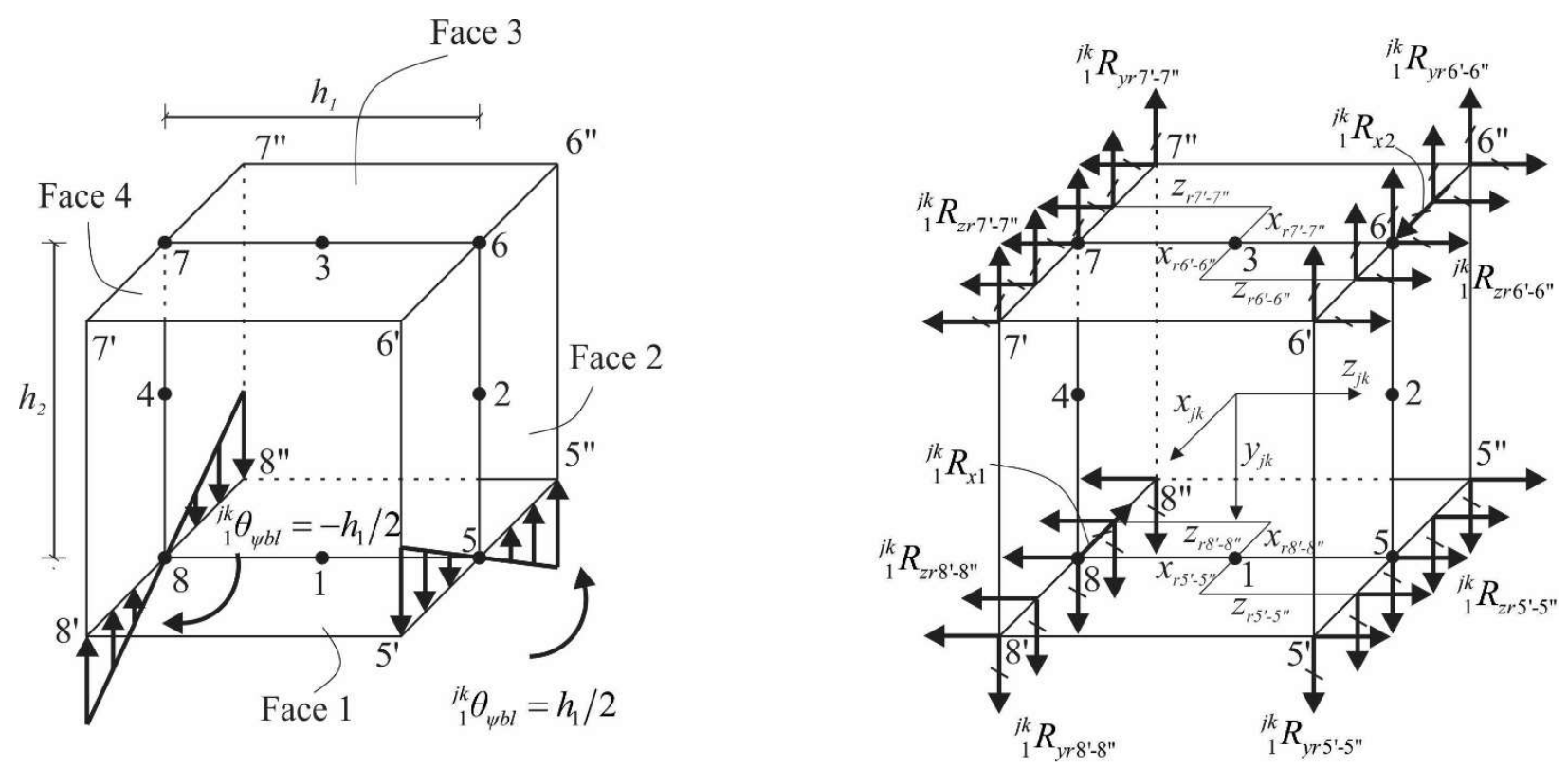

Fig. 7 (a) Unit warping deformation applied at Face 1; (b) boundary conditions and reactions

In Eq. 17, the warping function $\omega_{1}(s)$ illustrated in Fig. 5 is obtained by $\omega_{1}(s)= \pm\left(h_{1} / 2\right) x_{r}(s)$ along edges $5^{\prime}-5^{\prime \prime}$ and $8^{\prime}-8^{\prime \prime}$. Similarly, bimoments ${ }_{21}^{j k} B_{b l},{ }_{31}^{j k} B_{b l}$ and ${ }_{41}^{j k} B_{b l}$ induced at Faces 2-4, respectively, are determined from reactions through

$$
\begin{aligned}
& { }_{21}^{j k} B_{b l}=\int_{A_{1}} \sigma_{2} \omega_{2}(s) d A \approx \sum_{r=1}^{n r}\left({ }^{j k} R_{z r 5^{\prime}-5^{\prime \prime}}\right)\left(\frac{h_{2}}{2} x_{r 5^{\prime}-5^{\prime \prime}}\right)+\sum_{r=1}^{n r}\left({ }^{j k} R_{z r 6^{\prime}-6^{\prime \prime}}\right)\left(\frac{-h_{2}}{2} x_{r 6^{\prime}-6^{\prime \prime}}\right) \\
& { }_{31}^{j k} B_{b l}=\int_{A_{1}} \sigma_{3} \omega_{3}(s) d A \approx \sum_{r=1}^{n r}\left(R_{y r 6^{\prime}-6^{\prime \prime}}\right)\left(\frac{-h_{1}}{2} x_{r 6^{\prime}-6^{\prime \prime}}\right)+\sum_{r=1}^{n r}\left({ }^{j k} R_{y r 7^{\prime}-7^{\prime \prime}}\right)\left(\frac{h_{1}}{2} x_{r 7^{\prime}-7^{\prime \prime}}\right) \\
& { }_{41}^{j k} B_{b l}=\int_{A_{1}} \sigma_{4} \omega_{4}(s) d A \approx \sum_{r=1}^{n r}\left({ }^{j k} R_{z r 8^{\prime}-8^{\prime \prime}}\right)\left(\frac{-h_{2}}{2} x_{r 8^{\prime}-8^{\prime \prime}}\right)+\sum_{r=1}^{n r}\left({ }^{j k} R_{z r 7^{\prime}-7^{\prime \prime}}\right)\left(\frac{h_{2}}{2} x_{r 7^{\prime}-7^{\prime \prime}}\right)
\end{aligned}
$$

The following step is to subject the joint to a unit warping deformation ${ }_{2}^{j k} \psi_{b l}=1$ at Face 2 while setting to zero the warping deformations ${ }_{1}^{j k} \psi_{b l},{ }_{3}^{j k} \psi_{b l}$ and ${ }_{4}^{j k} \psi_{b l}$ at Faces 1, 3, and 4. The resulting reactions are extracted from the finite element model and the corresponding bimoments induced at Faces 1-4 are computed from the expressions given in Appendix B. The procedure is repeated by successively imposing unit warping deformation ${ }_{3}^{j k} \psi_{b l}=1,{ }_{4}^{j k} \psi_{b l}=1$, one at a time, at Faces 3 and 4 , while setting to zero the remaining warping deformations to obtain the corresponding bimoments induced at Faces 1 to 4 (Appendix B). 
The total bimoments ${ }_{1}^{j k} B_{b l}-{ }_{4}^{j k} B_{b l}$ acting on Faces 1-4 induced by warping deformations ${ }_{1}^{j k} \psi_{b l}-{ }_{4}^{j k} \psi_{b l}$ are obtained by superposition of Eqs. (17), (18a-c), (B1a-d), (B2a-d) and (B3a-d) yielding

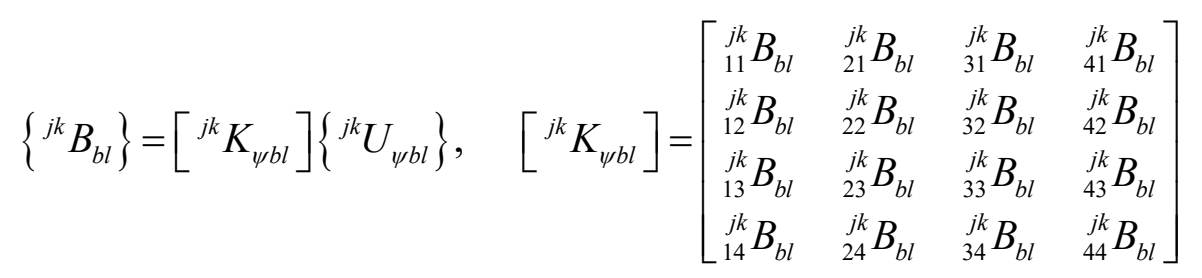

Eq. (19a-b) defines the warping matrix $\left[{ }^{j k} K_{\psi b l}\right]$ which relates the nodal bimoments $\left\langle{ }^{j k} B_{b l}\right\rangle^{T}=\left\langle{ }_{1}^{j k} B_{b l} \quad{ }_{2}^{j k} B_{b l} \quad{ }_{3}^{j k} B_{b l} \quad{ }_{4}^{j k} B_{b l}\right\rangle \quad$ to the nodal warping deformations $\left\langle{ }^{j k} U_{\psi b l}\right\rangle^{T}=\left\langle{ }_{1}^{j k} \psi_{b l} \quad{ }_{2}^{j k} \psi_{b l} \quad{ }_{3}^{j k} \psi_{b l} \quad{ }_{4}^{j k} \psi_{b l}\right\rangle$. The energy contribution of the warping matrices for the joints of the structure is

$$
\frac{1}{2} \delta^{2} \pi_{j}=\sum_{k=1}^{m} \frac{1}{2} \delta^{2}\left({ }^{j k} \pi_{k}\right)=\sum_{k=1}^{m} \frac{1}{2}\left\langle\delta\left({ }^{j k} U_{\psi b l}\right)\right\rangle_{1 \times 4}^{T}\left[{ }^{j k} K_{\psi b l}\right] 4 \times 4\left\{\delta\left({ }^{j k} U_{\psi b l}\right)\right\}_{4 \times 1}
$$

where the summation is over the number of joints $m$ in the structure.

\subsection{Special considerations for joints connecting fewer than four elements}

Eq. (19a-b) provides the general form of warping stiffness matrix $\left[{ }^{j k} K_{\psi b l}\right]$ for the case of a joint interfacing between four members. When a joint connects only two or three members, the bimoments acting on the unconnected faces of the joint vanish and static condensation is needed to formulate the warping stiffness matrices for such cases. It is expedient to re-order the warping degrees of freedom in Eq. (19a-b) so that the non-vanishing bimoments $\left\{B_{1}\right\}$ and corresponding warping deformations $\left\{\psi_{1}\right\}$ are grouped in the first partition and the vanishing bimoments $\left\{B_{2}\right\}$ and corresponding warping deformations $\left\{\psi_{2}\right\}$ are grouped in the second partition. The procedure is systematically achieved by introducing permutation matrices $[P]_{c}$ for various cases $c=1,2,3,4,5$ as defined in Table 1. Also, given in the table are the sizes of vectors $\left\{B_{1}\right\},\left\{\psi_{1}\right\}$, $\left\{B_{2}\right\}$, and $\left\{\psi_{2}\right\}$ for the joint configurations depicted in Fig. 2 and can be generalized to other cases. 
In all cases, the permutation matrices meet the condition $[P]_{c}[P]_{c}=[I]$ while re-arranging the degrees of freedom so that the vanishing bimoments are moved to bottom partition, i.e.,

$\left.[P]_{c}\left\{{ }^{j k} B_{b l}\right\}=\left\{\frac{\left\{B_{1}\right\}}{\left\{B_{2}\right\}}\right\},[P]_{c}\left\{{ }^{j k} U_{\psi b l}\right\}=\left\{\frac{\left\{\psi_{1}\right\}}{\left\{\psi_{2}\right.}\right\}\right\}$

By pre-multiplying Eq. (19a) by $[P]_{c}$ and inserting $[P]_{c}[P]_{c}=[I]$ in the right hand side term, one obtains

$[P]_{c}\left\{{ }^{j k} B_{b l}\right\}=[P]_{c}\left[{ }^{j k} K_{\psi b l}\right][P]_{c}[P]_{c}\left\{{ }^{j k} U_{\psi b l}\right\}$, or

$\left\{\begin{array}{l}\left\{B_{1}\right\} \\ \hdashline\left\{B_{2}\right\}\end{array}\right\}=\left[\begin{array}{l:l}{\left[K_{11}\right]} & {\left[K_{12}\right]} \\ \hdashline\left[K_{12}\right]^{T} & {\left[K_{22}\right]}\end{array}\right]\left\{\begin{array}{l}\left\{\psi_{1}\right\} \\ \hdashline\left\{\psi_{2}\right\}\end{array}\right\}, \quad\left[\begin{array}{c:c}{\left[K_{11}\right]} & {\left[K_{12}\right]} \\ \hdashline\left[K_{12}\right]^{T} & {\left[K_{22}\right]}\end{array}\right]=[P]_{c}\left[{ }^{j k} K_{\psi b l}\right][P]_{c}$

By setting $\left\{B_{2}\right\}=\{0\}$, expanding Eq. (22a-b) along the second partition, and solving for $\left\{\psi_{2}\right\}$, one obtains

$\left\{\psi_{2}\right\}=-\left[K_{22}\right]^{-1}\left[K_{12}\right]^{T}\left\{\psi_{1}\right\}$

By expanding the first partition of Eq. (22a-b) and substituting from Eq. (23), one obtains

$\left(\left[K_{11}\right]-\left[K_{12}\right]\left[K_{22}\right]^{-1}\left[K_{12}\right]^{T}\right)\left\{\psi_{1}\right\}=\left\{B_{1}\right\}$

where the bracketed term represents the modified stiffness matrix sought which accounts for the condition $\left\{B_{2}\right\}=\{0\}$. The numeric values of the warping matrices obtained for various joint configurations in the examples investigated in the present study are provided in Appendix C. 
Table 1 Permutation matrices and sizes of nodal bimoment and warping vectors for various joint configurations

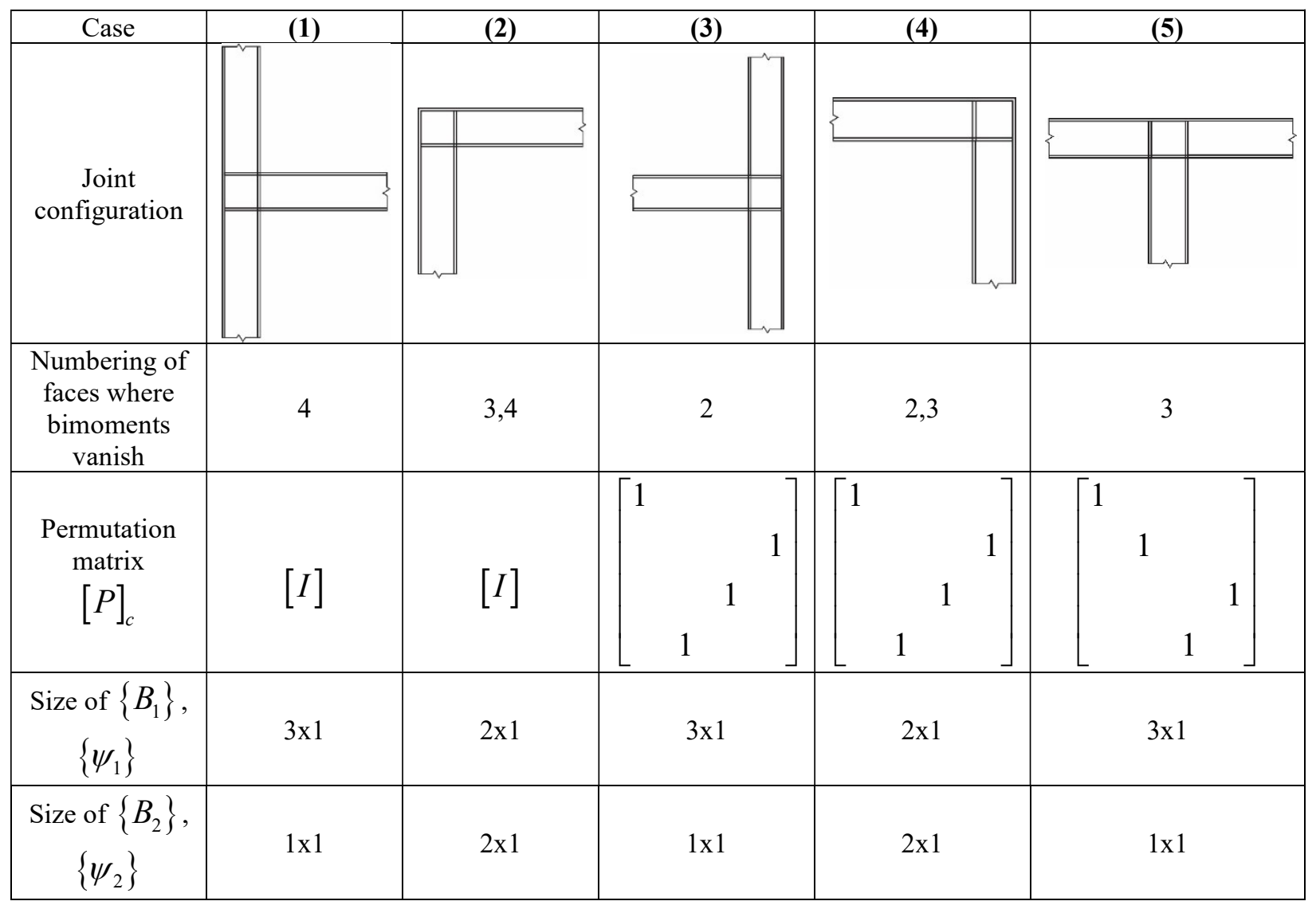

\subsection{Potential energy gain due to non-conservative moments}

Consider the four-nodded joint element $k$ as a part of 2D frame shown in Fig. 8a. Under reference loads (Configuration 2 in Fig. 3) the joint is in equilibrium and the sum of moments $M$ at faces $i=1, \ldots, 4$ must vanish, i.e.,

$\sum_{i=1}^{4}{ }_{i}^{j k} M=0 \Rightarrow{ }_{1}^{j k} M=-{ }_{2}^{j k} M-{ }_{3}^{j k} M-{ }_{4}^{j k} M$

At the onset of buckling (Configuration 3 in Fig. 3), this equilibrium equation takes the form of

$$
\sum_{i=1}^{4} \lambda\left({ }_{i}^{j k} M\right)=0
$$


Moments ${ }_{i}^{j k} M(i=1, \ldots, 4)$ are depicted as a couple of tensile and compressive forces ${ }_{i}^{j k} F_{3}$ (Fig. 8 b) applied at Faces 1 to 4 where the right subscript takes the value 3 to denote Configuration 3 (Fig. 3) at the onset of buckling. When the structure buckles, the joint undergoes rotations $\theta_{y b}$ and $\theta_{z b}$. If the moments rotate with the joint (non-conservative moments), the point of application of forces ${ }_{i}^{j k} F_{3}$ move by a distance $(d / 2){ }^{j k} \theta_{y b}$ and thus induces a second order weak-axis moment $\lambda{ }_{i}^{j k} F_{3} d{ }^{j k} \theta_{y b}$ as shown in Fig. 8c. When the joint undergoes buckling rotation ${ }^{j k} \theta_{z b}$, the weak-axis moment $\lambda{ }_{i}^{j k} F_{3} d{ }^{j k} \theta_{y b}$ induces a load potential gain $\lambda\left({ }_{i}^{j k} M\right)\left({ }^{j k} \theta_{y b}\right)\left({ }^{j k} \theta_{z b}\right)$ (Fig. 8d). The second order moments induced about y-axis and z-axis on all faces are respectively shown in Fig. 8e and Fig. 8f. Subscripts 4 for forces $\lambda{ }_{i}^{j k} F_{4}$ denote the equivalent forces at Configuration 4 in Fig. 3 (i.e., at the buckled configuration). The load potential gained by the second order moments at all four faces due to the joint undergoing $\theta_{y b}$ and $\theta_{z b}$ is $V_{F R}=\lambda\left(-{ }_{1}^{j k} M+{ }_{2}^{j k} M-{ }_{3}^{j k} M+{ }_{4}^{j k} M\right){ }^{j k} \theta_{y b}{ }^{j k} \theta_{z b}$

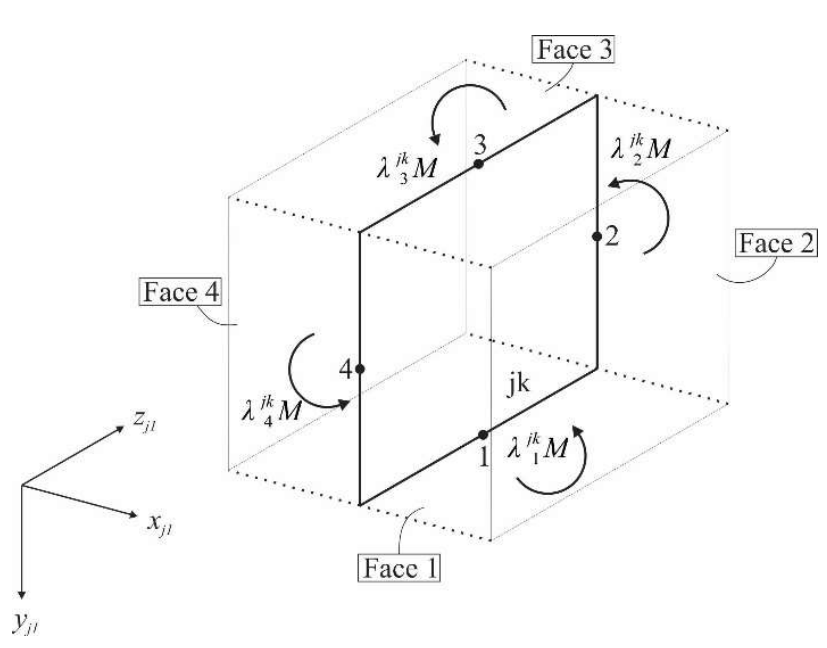

(a)

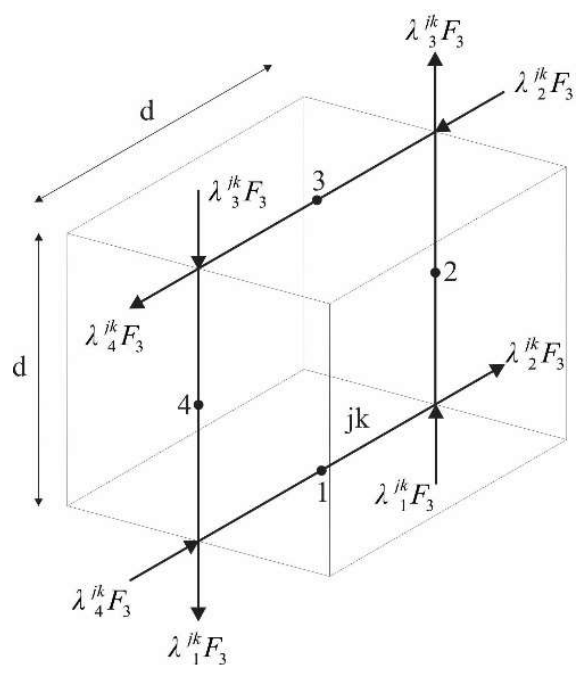

(b) 


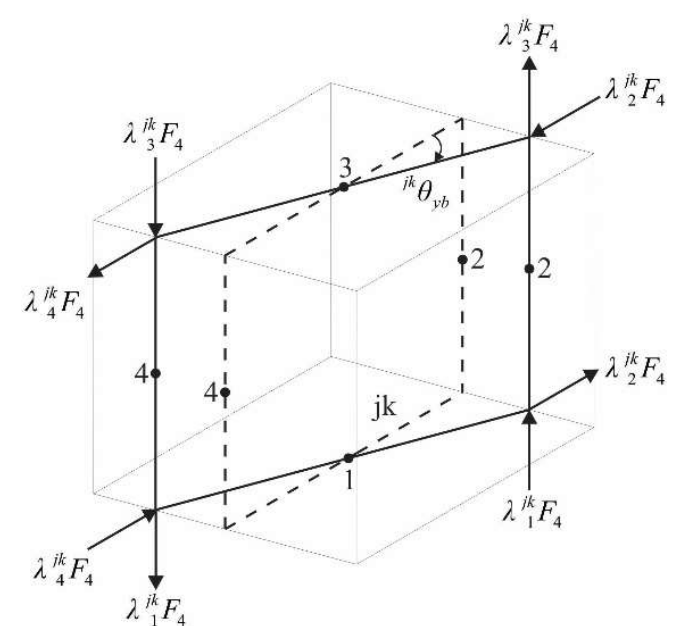

(c)

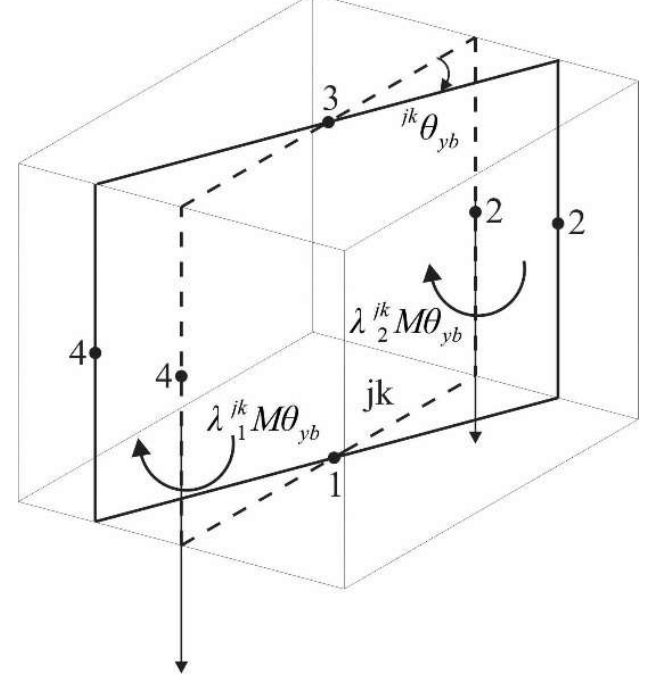

(e)

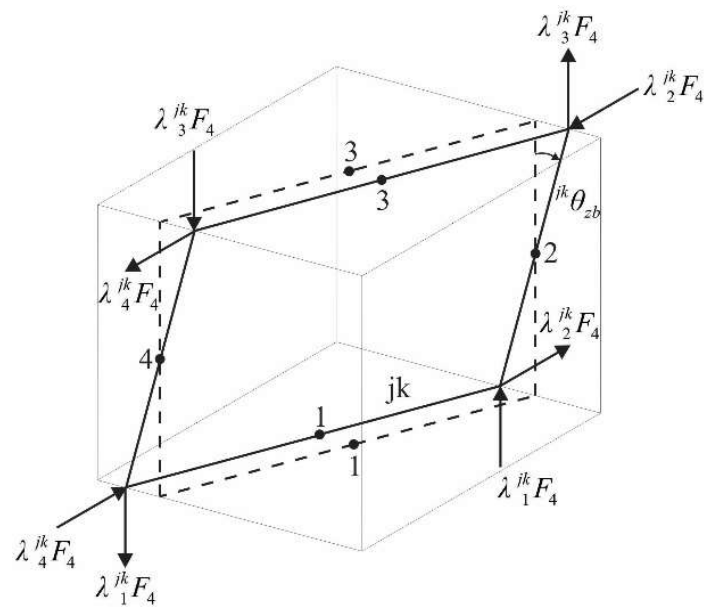

(d)

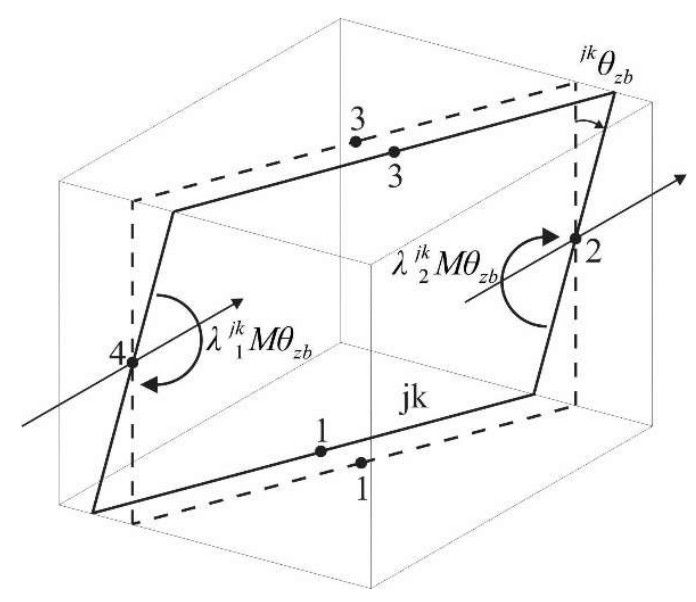

(f)

Fig. 8 (a) Joint element under moments at the onset of buckling, (b) Joint element with equivalent force couples, (c) Joint after rotation about weak-axis, (d) Final position of the joint after rotation about z-axis, (e) Induced weak-axis moments, and (f) Second order moments due to rotation

Considering the special case where only two collinear members are connected to faces 1 and 3, Eqs. (25) and (27) lead to the simplification

$$
V_{F R}=\lambda\left(-{ }_{1}^{j k} M-{ }_{3}^{j k} M\right){ }^{j k} \theta_{y b}{ }^{j k} \theta_{z b}=\lambda\left({ }_{3}^{j k} M-{ }_{3}^{j k} M\right){ }^{j k} \theta_{y b}{ }^{j k} \theta_{z b}=0
$$

Thus, for collinear members, the contribution potential energy gain due to non-conservative moments vanishes. Unlike the general case of a joint connected to four elements where the omission of joint rotation effects destroys joint equilibrium, for collinear members, the omission of joint rotation does not disturb the joint equilibrium and the omission of joint rotation effect still 
leads to a correct buckling solution. This approach was successfully used in various collinear solutions (e.g., [5], [7], [10] and [14]).

Considering the case of a joint $k$ connecting two perpendicular members at Faces 1 and 2. Unlike the case of collinear members, the potential energy gain induced by second order moments due moments ${ }_{1}^{j k} M$ and ${ }_{2}^{j k} M$ will not cancel one another and the load potential energy gain due to joint rotation is

$V_{F R}=\lambda\left(-{ }_{1}^{j k} M+{ }_{2}^{j k} M\right){ }^{j k} \theta_{y b}{ }^{j k} \theta_{z b} \neq 0$

In such a case, the incorporation of joint rotation effect becomes essential for the proper prediction of the critical load of the frame.

\subsection{Condition of Neutral Stability for the Structure}

The structure stiffness matrix $[K]_{s}$, reflecting the stiffness contributions of the members, the warping stiffness contributions of joints, and the structure geometric stiffness $\left[K_{G}\right]_{S}$ are formed using conventional assembly technique. From the kinematic conditions in Eqs. (16), by substituting into Eq. (1), one obtains the variational statement for the structure

$$
\delta\left(\frac{1}{2} \delta^{2} \pi\right)=\delta\left\{\frac{1}{2}\left\langle\delta u_{s}\right\rangle^{T}\left([K]_{s}+\lambda\left[K_{G}\right]_{s}\right)\left\{\delta u_{s}\right\}+\left\langle\delta u_{s}\right\rangle^{T}[B]\{\delta L\}\right\}=0
$$

in which $\left\{u_{s}\right\}$ is the vector nodal displacements for the structure. By evoking the stationarity conditions $\partial\left(1 / 2 \delta^{2} \pi\right) / \partial\left\{\delta u_{s}\right\}=\{0\}, \partial\left(1 / 2 \delta^{2} \pi\right) / \partial\{\delta L\}=\{0\}$, one recovers the following eigenvalue problem in the unknown load multiplier $\lambda$

$$
\left(\left[\begin{array}{cc}
{[K]_{s}} & {[B]^{T}} \\
{[B]} & {[0]}
\end{array}\right]+\lambda\left[\begin{array}{cc}
{\left[K_{g}\right]_{s}} & {[0]} \\
{[0]} & {[0]}
\end{array}\right]\right)\left\{\begin{array}{l}
\left\{u_{s}\right\} \\
\{L\}
\end{array}\right\}=\{0\}
$$




\section{Examples}

\subsection{Example 1: $\Gamma$-shaped frame}

A laterally unsupported $\Gamma$-shaped frame (Fig. 9a) consisting of a column of height $L_{c}=4000 \mathrm{~m}$ and a horizontal cantilever of span $L_{b}=0.8 \mathrm{~m}$ connected through a moment connection consisting of two pairs of stiffeners. The frame is subjected to a gravity load $P$ acting the shear center at the tip of the cantilever. The frame is assumed to remain within its elastic range of deformation. The cross-section profile is W200x59, which meets the compactness requirements in ANSI/AISC 36016 for a yield strength of $350 \mathrm{MPa}$. The sectional properties for the idealized cross section (i.e., while neglecting the fillets) rather than the real sections were adopted be consistent with the shell finite element analysis model, which does not model the fillets. The idealized cross-sectional properties are provided in Fig. 9b. It is required to determine the elastic lateral buckling load of the frame based on various solutions, including the one developed in the current study by considering the effect of joint rotations.

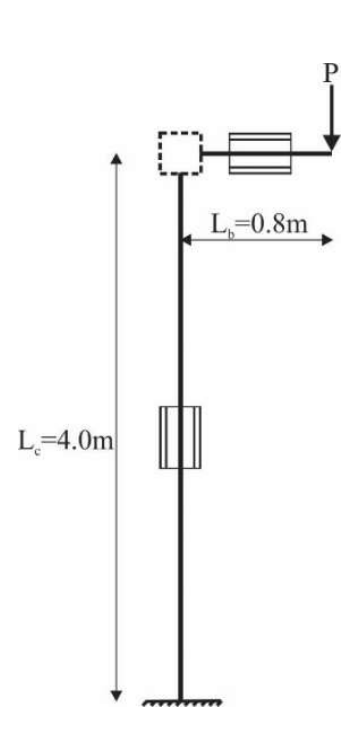

(a)

$\begin{array}{ll}\text { Width } b(\mathrm{~mm}) & 205.0 \\ \text { Height } h(\mathrm{~mm}) & 195.8 \\ \text { Flange thickness } t(\mathrm{~mm}) & 14.20 \\ \text { Web thickness } w(\mathrm{~mm}) & 9.100 \\ \text { Principal moment of inertia } I_{x}\left(\mathrm{~mm}^{4}\right) & 61.32 \times 10^{6} \\ \text { Principal moment of inertia } I_{y}\left(\mathrm{~mm}^{4}\right) & 20.10 \times 10^{6} \\ \text { Torsional constant } J\left(\mathrm{~mm}^{4}\right) & 4.390 \times 10^{5} \\ \text { Warping constant } I_{\omega}\left(\mathrm{mm}^{6}\right) & 1.930 \times 10^{11} \\ \text { Area } A\left(\mathrm{~mm}^{2}\right) & 7575\end{array}$

(b)

Fig. 9 -shaped frame for Example 1 (a) Geometry and (b) Sectional properties for W200x59 section 
Seven solutions were conducted and summarized in Table 2. The number of elements used in each model is reported to provide an indication of the computational effort for each analysis. Solution 1 is based on a shell element analysis using the S4R element in ABAQUS. Solution 2 is based on the joint formulation developed herein in conjunction with the shear deformable elements of Sahraei and Mohareb [14] to represent the beam and columns. Solution 3 is based on the ABAQUS shear deformable B31OS element which captures shear deformation due to bending but omits shear deformation due to warping [14]. Solution 4 is based on the present joint element in conjunction with the classical element by Barsoum and Gallagher [5]. Solution 5 is based on shear deformable elements while assuming the warping deformation is continuous (in line with the solutions developed in Mohareb and Dabbas [44], Zinoviev and Mohareb [45], Rizzi and Varano [51], and Basaglia et al. [47]). Solution 6 is based on a rigid representation of the joint (by assuming zero warping deformation at the column top and beam left end) in conjunction with shear deformable elements, while Solution 7 is based on a rigid representation of the joint in conjunction with classical beam elements. The rigid joint representation adopted in Solutions 6 and 7 would be approached only if the joint had three pairs of stiffeners including a diagonal pair of stiffeners [52] and its use in the present problem with two pairs of stiffeners providing only partial warping restraints is expected to overestimate buckling loads.

Given the large number of degrees of freedom involved in the shell model in Solution 1, the corresponding buckling load prediction $P_{c r 1}=170.9 \mathrm{kN}$ provides the most accurate prediction. The critical load $P_{c r 1}$ is used as a reference value against which other buckling predictions are compared. Solution 2, based on the joint element developed in the present study, and including joint rotation effects, provides a very close prediction of the buckling load, only $1.2 \%$ higher than that based on the shell solution at a fraction of the computational cost. The difference is attributed to distortional effects, captured in the shell solution but not in the SM-M element. If the joint rotation effect is excluded from Solution 2, the predicted critical load is found to be $231.1 \mathrm{kN}$, grossly overestimating the buckling load by $35 \%$ compared to Solution 1 . The comparison showcases the importance of incorporating the joint rotation effect in the formulation. 
Table 2 Critical loads $(\mathrm{kN})$ for gamma-shaped frame based on different solutions

\begin{tabular}{|c|c|c|c|c|}
\hline \multicolumn{3}{|c|}{ Solution type } & \multirow{2}{*}{$\begin{array}{l}\text { Critical } \\
\text { load } \\
(\mathbf{k N})\end{array}$} & \multirow{2}{*}{$\begin{array}{l}\text { Critical } \\
\text { load } \\
\text { relative } \\
\text { to } P_{c r 1} \\
\end{array}$} \\
\hline$P_{c r}$ & Member representation & Joint representation & & \\
\hline$P_{c r 1}$ & $\begin{array}{c}\text { Shell (S4R)-finite element } \\
\text { analysis solution (column: } 2400 \\
\text { elements; beam: } 480 \text { elements) }\end{array}$ & $\begin{array}{l}320 \text { shell-finite element } \\
\text { analysis solution (S4R) }\end{array}$ & 170.9 & ---- \\
\hline$P_{c r 2}$ & $\begin{array}{l}\text { SM-M element [14] fully } \\
\text { deformable in shear (column: } 20 \\
\text { elements; beam } 4 \text { elements) }\end{array}$ & $\begin{array}{l}\text { Present joint formulation } \\
\text { Including joint rotation effects }\end{array}$ & 173.0 & 1.012 \\
\hline$P_{c r 3}$ & $\begin{array}{l}\text { ABAQUS B31OS beam element } \\
\text { (shear deformable in bending but } \\
\text { not in warping; column: } 20 \\
\text { elements; beam } 10 \text { elements) }\end{array}$ & $\begin{array}{l}\text { Treated as a two-noded joint } \\
\text { connected with rigid multi- } \\
\text { point constraints and different } \\
\text { warping deformations }\end{array}$ & 173.1 & 1.013 \\
\hline$P_{c r 4}$ & $\begin{array}{c}\text { Classical non-shear deformable } \\
\text { element [5] (column: } 20 \\
\text { elements; beam } 4 \text { elements) }\end{array}$ & $\begin{array}{l}\text { Present joint formulation } \\
\text { Including joint rotation effects }\end{array}$ & 176.8 & 1.035 \\
\hline$P_{c r 5}$ & $\begin{array}{l}\text { SM-M [14] element fully } \\
\text { deformable in shear (column: } 20 \\
\text { elements; beam } 4 \text { elements) }\end{array}$ & $\begin{array}{l}\text { Continuous warping } \\
\text { deformation }\end{array}$ & 178.1 & 1.042 \\
\hline$P_{c r 6}$ & $\begin{array}{l}\text { SM-M [14] element fully } \\
\text { deformable in shear (column: } 20 \\
\text { elements; beam } 4 \text { elements) }\end{array}$ & $\begin{array}{l}\text { Rigid joint representation with } \\
\text { full warping restraint }\end{array}$ & 209.8 & 1.228 \\
\hline$P_{c r 7}$ & $\begin{array}{c}\text { Classical non-shear deformable } \\
\text { element [5] (column: } 20 \\
\text { elements; beam } 4 \text { elements) }\end{array}$ & $\begin{array}{l}\text { Rigid joint representation with } \\
\text { full warping restraint }\end{array}$ & 210.4 & 1.231 \\
\hline
\end{tabular}

Solution 3 provides a critical load estimate based on the ABAQUS thin-walled shear deformable B31OS element while modeling the joint using two nodes rigidly connected using multi-point constraints. The comparison shows almost identical results to Solution 2 (This conclusion is not universal as will be shown in the following example). Similar to the SM-M element, B31OS element does not capture distortional effects and thus predicts higher critical moment predictions compared to that of S4R shell solution. Solution 4 based on the classical non-shear deformable element overestimates the buckling load by $3.5 \%$ compared to Solution 1 . The difference is caused by the fact that the classical solution captures neither distortional nor shear deformation effects. The influence of the method of representation of the joint is assessed by comparing Solutions 2 and 6, in which a rigid jointed representation with full warping restraints (Solution 6) is found to over-predict the buckling load by about $21.6 \%$ compared to Solution 2 based on the more realistic representation of the joint introduced in the present study. When warping deformation is assumed continuous by equating the warping deformation at the column top to that at the cantilever left end 
(Solution 5), the solution was found to over-predict the buckling load by $4.2 \%-1.2 \%=3.0 \%$ compared to Solution 2. The comparison suggests that the joint formulation developed in the current study provides the most flexible representation of the joint flexibility and thus yields the closest results compared to the shell element solution (Solution 1). The continuous warping deformation assumption is found to lead to a stiffer model, thus predicting a higher critical load, but still provides the second best representation of the joint, while the rigid joint representation grossly overestimates the stiffness provided by the joint. A similar conclusion is reached when comparing Solutions 4 and 7, both based on the classical element, in which Solution 7 based on a rigid joint representation is found to overestimate the buckling load by $23.1-3.5=19.6 \%$ compared with Solution 4 (based on a the present flexible joint representation).

The scenario where the beam is separated from the frame (thus omitting the flexibility of the column at the cantilever root) is investigated by conducting a buckling analysis of the beam alone with 8 fully shear deformable elements [12]. The obtained critical load of 9,980 kN grossly overestimates the buckling load compared to other solutions which account for beam-column interaction. Contrary to the widely accepted proposition by Salvadori [3], separating the member from the whole frame and assuming idealized end boundary conditions, leads to un-conservative critical moment predictions in the present frame.

\subsection{Example 2: Single story portal frame}

The portal frame studied in [45] is re-investigated. Column heights are $L_{c}=4 m$ and beam span is $L_{b}=6 m$. Cross-sections are W200x59 (Compact section as described in Example 1). Columns are fully fixed at the bases (Fig. 10a) while the rest of frame is laterally unsupported out of the plane of the frame as no lateral restraints are provided at the beam to column junction. Two point loads $P$ are acting at third-span points. The relevant idealized cross-section properties have been provided in Fig. 9b. The elastic lateral buckling load for the frame is sought. It is considered that the (a) yield strength of the frame is high enough so that the capacity of the frame is not governed by yielding considerations. 


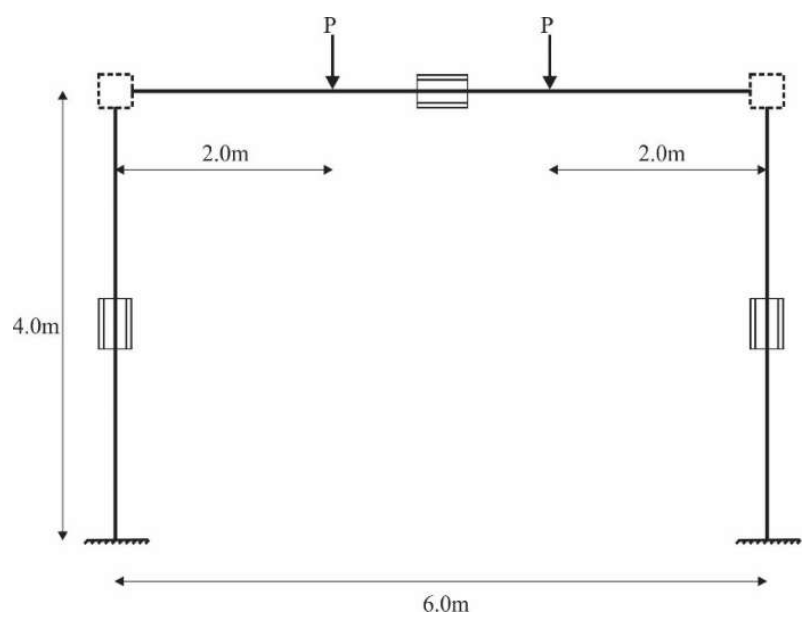

(a)

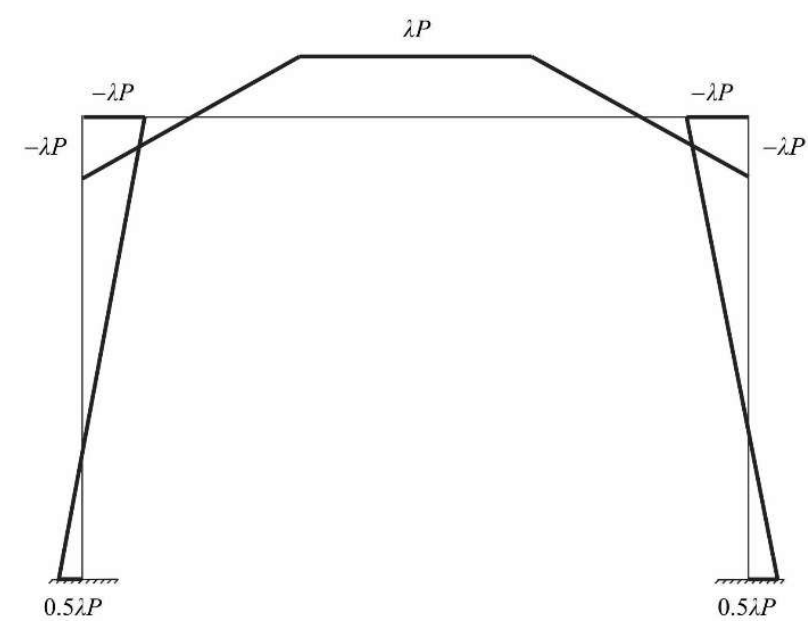

(b)

Fig. 10 Portal frame (a) geometry and reference loading, and (b) Bending moment diagram at onset of bucking

The bending moment diagram under the applied reference loads is shown in Fig. 10b. In a manner similar to example 1, seven solution are conducted to predict the critical moment of the portal frame. The number of elements adopted in each solution are provided in Table 3. Solution 1 based on the shell analysis provides the lowest critical load prediction $P_{c r 1}$ and is taken as a reference against which the remaining solutions are compared. The buckling load prediction of Solution 2 (based on the present joint formulation including joint rotation effects and shear deformable elements) is found to be $5.8 \%$ higher than that based on the shell FEA and is the closest to the shell solution. The difference between both solutions is higher than in Example 1, suggesting that distortional effects for the portal frame are more significant than the Gamma-shaped frame. When the joint rotation effect is omitted, the critical load is found to increase to $244.7 \mathrm{kN}$, thus overpredicting the buckling load by $11.4 \%$ compared to Solution 1 . The effect of joint rotation results in an $11.4 \%-5.8 \%=6.4 \%$ over-prediction of the critical load, which is significantly lower than the $35 \%$ difference observed for the Gamma-shaped frame in example. The large difference from the joint rotation effect observed in example 1 is attributed to the fact that the column in example 1 undergoes significant twist throughout LTB compared to that in example 2, thus increasing the contribution of the destabilizing term $\lambda\left(-{ }_{1}^{j k} M+{ }_{2}^{j k} M\right){ }^{j k} \theta_{y b}{ }^{j k} \theta_{z b}$ arising in Eq. (29).

Solution 3 overestimates the critical load by $P_{c r 3} / P_{c r 1}-P_{c r 2} / P_{c r 1}=1.117-1.058=5.9 \%$ compared to Solution 2. As discussed in Example 1, the difference is due to shear deformation effects induced 
by warping. Solution 4 fully omits shear deformation in members and is observed to predict a higher buckling load compared to Solution 2 which captures shear deformation. The difference between both solutions is $P_{c r 4} / P_{c r 1}-P_{c r 2} / P_{c r 1}=1.131-1.058=7.3 \%$, which is larger than the $2.3 \%$ difference observed in Example 1, suggesting that shear deformation has a more pronounced effect for the portal frame. The finding is consistent with the fact that a portal frame, with two fixed supports, is more constrained than the $\Gamma$-shaped frame with a single support at the base, thus increasing the effect of shear deformation.

Solutions 5 based on continuous warping restraint is observed to over-predict the buckling load by $P_{c r 5} / P_{c r 1}-P_{c r 2} / P_{c r 1}=1.151-1.058=9.3 \%$ compared to Solution 2 which adopts the present joint formulation. The 9.3\% difference is larger than 3.0\% difference in Example 1 since the warping stiffness and kinematic relations for the two joints involved in Example 2 is more pronounced compared to Example 1 where only one joint was modeled through kinematic relations.

A rigid joint representation with shear deformable elements (Solution 6) and with non-shear deformable elements (solution 7) are observed to over-predict the buckling load by $P_{c r 6} / P_{c r 1}-P_{c r 2} / P_{c r 1}=1.202-1.058=14.4 \%$ and $P_{c r 7} / P_{c r 1}-P_{c r 2} / P_{c r 1}=1.316-1.058=25.8 \%$,

respectively, compared to Solution 2. Both results illustrate (a) the importance of accounting for joint flexibility and (b) shear deformation effects are more pronounced in the present example compared to the corresponding percent differences of $21.6 \%$ and $21.9 \%$ obtained in for Solutions 6 and 7 of Example 1.

Adopting the present standard methodology $[1,2]$ of isolating members from the rest of structure and quantifying their critical moments separately, the horizontal member is separated from the rest of the portal frame and subjected to the two point loads and the end moments as obtained from the pre-bucking analysis shown in Fig. 11b. Idealized pinned boundary conditions are assumed for the vertical displacement, lateral displacement, and angle of twist. Based on ANSI/AISC 360-16, the moment gradient factor is $C_{A I S C}=12.5 M_{\text {max }} /\left(2.5 M_{\text {max }}+3 M_{A}+4 M_{B}+3 M_{C}\right)$ where $M_{A}, M_{B}$, and $M_{C}$ are the quarter point moments, and $M_{\max }$ is the peak moment, yielding $C_{A I S C}=1.316$, and the corresponding critical moment is $M_{c r-A I S C}=C_{A I S C}\left(\pi / L_{u}\right) \sqrt{E I_{y} G J+\left(\pi E / L_{u}\right)^{2} I_{y} C_{w}}=291 \mathrm{kNm}$. Under the Canadian standards CAN/CSA S16-14, the moment gradient factor is given by 
$C_{C A N}=4 M_{\max } / \sqrt{M_{\text {max }}^{2}+4 M_{A}^{2}+7 M_{B}^{2}+4 M_{C}^{2}}=1.265$, yielding a critical moment prediction $M_{c r-C A N}=C_{C A N}\left(\pi / L_{u}\right) \sqrt{E I_{y} G J+\left(\pi E / L_{u}\right)^{2} I_{y} C_{w}}=280 \mathrm{kNm}$. For the buckled member, the corresponding axial force in the member at the onset under reference load $C_{f}=0.374 \lambda=0.374 \times 232=89.6 \mathrm{kN}$ is relatively small compared to the buckling strength $C_{r y}=1102 k N$ of the column (i.e., $C_{f} / C_{r y}=0.08$ ) suggesting that the buckling strength of the member is predominantly induced by strong-axis bending. Standard-based elastic critical moment predictions based on both standards are found to over-predict the critical moment based on the shell analysis $(219.7 \mathrm{kNm})$ and the present solution. This is the case since the quarter point moment gradient equations are only approximate and are intended for beam segments that are restrained laterally and torsionally but free to warp and undergo lateral rotation at both ends. Such idealized conditions are not representative of the end conditions of the horizontal member where both ends are partially restrained relative to the lateral displacement, twist, warping, and weak-axis rotation through the adjoining columns. The comparison suggests the importance of accounting for interaction effects between the beam and columns as is the case in ABAQUS shell solutions and the present solutions.

Table 3 Critical Loads (kN) for single story portal frame

\begin{tabular}{|c|c|c|c|c|}
\hline \multicolumn{3}{|c|}{ Solution type } & $\begin{array}{c}\text { Critical } \\
\text { load } \\
\text { (kN) }\end{array}$ & $\begin{array}{c}\text { Critical } \\
\text { load } \\
\text { relative } \\
\text { to } \boldsymbol{P}_{c r 1}\end{array}$ \\
\hline$P_{c r}$ & Member representation & Joint representation & ---- \\
\hline$P_{c r 1}$ & $\begin{array}{c}\text { Shell (S4R)-finite element } \\
\text { analysis solution (column: 2400 } \\
\text { elements; beam: 3600 elements) }\end{array}$ & $\begin{array}{c}320 \text { shell-finite element } \\
\text { analysis solution (S4R) }\end{array}$ & 219.7 & 1.058 \\
\hline$P_{c r 2}$ & $\begin{array}{c}\text { SM-M element [14] fully } \\
\text { deformable in shear (column: 20 } \\
\text { elements; beam 30 elements) }\end{array}$ & $\begin{array}{c}\text { Present joint formulation } \\
\text { Including joint rotation effects }\end{array}$ & 232.4 & 1.117 \\
\hline$P_{c r 3}$ & $\begin{array}{c}\text { ABAQUS B31OS beam element } \\
\text { (shear deformable in bending but } \\
\text { not in warping; column: 20 } \\
\text { elements; beam 30 elements) }\end{array}$ & $\begin{array}{c}\text { Treated as a two-noded joint } \\
\text { connected with rigid multi- } \\
\text { point constraints and different } \\
\text { warping deformations }\end{array}$ & 245.4 & 1.131 \\
\hline$P_{c r 4}$ & $\begin{array}{c}\text { Classical [5] non-shear } \\
\text { deformable element (column: 20 } \\
\text { elements; beam 30 elements) }\end{array}$ & $\begin{array}{c}\text { Present joint formulation } \\
\text { Including joint rotation effects }\end{array}$ & 248.4 & 252.8 \\
\hline$P_{c r 5}$ & $\begin{array}{c}\text { SM-M [14] element fully } \\
\text { deformable in shear (column: 20 } \\
\text { elements; beam 30 elements) }\end{array}$ & $\begin{array}{c}\text { Continuous warping } \\
\text { deformation }\end{array}$ & 1.151 \\
\hline
\end{tabular}




\begin{tabular}{|c|c|c|c|c|}
\hline$P_{c r 6}$ & $\begin{array}{c}\text { SM-M [14] element fully } \\
\text { deformable in shear (column: 20 } \\
\text { elements; beam 30 elements) }\end{array}$ & $\begin{array}{c}\text { Rigid joint representation with } \\
\text { full warping restraint }\end{array}$ & 264.1 & 1.202 \\
\hline$P_{c r 7}$ & $\begin{array}{c}\text { Classical [5] non-shear } \\
\text { deformable element (column: 20 } \\
\text { elements; beam 30 elements) }\end{array}$ & $\begin{array}{c}\text { Rigid joint representation with } \\
\text { full warping restraint }\end{array}$ & 289.2 & 1.316 \\
\hline
\end{tabular}

\subsection{Example 3: Three-story single-bay frame}

The three-storey single-bay frame shown in Fig. 11a is fixed at bases $A$ and $B$, and subjected to the shown uniformly distributed gravity load applied to the third-story beam. All members are assumed to have W200x46 cross-section (Compact section for a yield strength of $350 \mathrm{MPa}$ ) and connected through moment resisting connections. Cross-sectional properties of W200x46 are: $b=203.0 \mathrm{~mm}, \quad h=192.0 \mathrm{~mm}, \quad t=11 \mathrm{~mm}, \quad w=7.2 \mathrm{~mm}, \quad I_{x}=4.545 \times 10^{7} \mathrm{~mm}^{4}$, $I_{y}=1.534 \times 10^{7} \mathrm{~mm}^{4}, \quad J=2.040 \times 10^{5} \mathrm{~mm}^{4}, \quad I_{\omega}=1.413 \times 10^{11} \mathrm{~mm}^{6}$, and $A=5848 \mathrm{~mm}^{2}$. It is required to determine the elastic LTB resistance of the frame based on two scenarios: (a) All beamcolumn junctions are restrained against lateral displacement (as is commonly the case in conventional buildings), and (b) No lateral restraints are provided at the beam-column junction except junctions $\mathrm{E}$ and $\mathrm{F}$ (as may be the case in some pipe rack configurations in industrial plants). The yield strength of steel is assumed to be high enough so that the resistance of the frame is governed by its elastic lateral torsional buckling resistance.

The bending moment and normal force diagrams under the reference load based on a pre-buckling analysis are provided in (Fig. 11b-c). The critical load combination of the whole three-story frame was determined for the laterally restrained and unrestrained scenarios using three solutions: (1) A shell finite-element analysis based on the S4R element in ABAQUS; (2) Based on the joint element developed under the present solution in conjunction with shear deformable beam elements (based on Sahraei and Mohareb [14]) to model the straight segments; and (3) Based on the B31OS element in ABAQUS with an assumed continuous warping restraint at the joints. The critical load multipliers $\lambda$ predicted by the three analyses are summarized in Table 4 . As expected, Solution 1 predicts the smallest critical load since it provides the most flexible representation of the structure. The corresponding buckling load multiplier is considered as a benchmark to assess the validity of other two solutions. Using the joint element developed in the current study with the shear deformable beam elements leads to a closer buckling prediction to the shell solution when compared to that based on the B31OS element with continuous warping at the joints which tend 
to overestimate the buckling resistance of frame. The present solution overestimates the buckling strength of the frame by $2 \%$ for the laterally restrained frame and by $7 \%$ for the case of no lateral restraint. The number of degrees of freedom involved in the present study is two orders of magnitudes less than that of the shell analysis. As expected, the present solution provides a reasonably accurate representation of the joint behaviour and the beam to column junctions. Figure 11 depicts the buckling configurations for both scenarios based on the ABAQUS simulation. The top beam deforms the most and thus the frame global buckling is attributed predominantly to the top beam.

By restraining the frame at all the junctions (Scenario (a)), Solutions 1-3 yield considerably higher buckling load predictions compared to Scenario (b) where lateral restraints are provided only at junctions $\mathrm{E}$ and F. For instance, the present solution predicts a peak critical moment of $315.3 \mathrm{kNm}$ for Scenario (a) and only 125.2kNm under Scenario (b). As observed in Fig. 11b, the peak bending moment occurs at mid-span of beam GH. In line with the standards procedure, member GH beam is isolated from the structure and its critical moment is determined from ANSI/AISC 360-16. The corresponding moment gradient factor is $C_{A I S C}=1.285$, and the corresponding critical moment is $M_{c r-A I S C}=231.8 \mathrm{kNm}$. According to $\mathrm{CAN} / \mathrm{CSA} \mathrm{S} 16-14$, the moment gradient factor is $C_{C A N}=1.246$ and the corresponding critical moment is $M_{c r-C A N}=224.8 \mathrm{kNm}$. Both values are lower than the critical moment of the braced frame (a), suggesting that the common standard practice of isolating the member from the structure based on the Salvadori hypothesis [3] leads to conservative predictions of the critical moments. In contrast, for the laterally unbraced frame (b), the procedure is found to overestimate the lateral torsional buckling resistance of the member. The example suggests restricting the adoption of the Salvadori hypothesis to structures where members are laterally braced at both ends. 


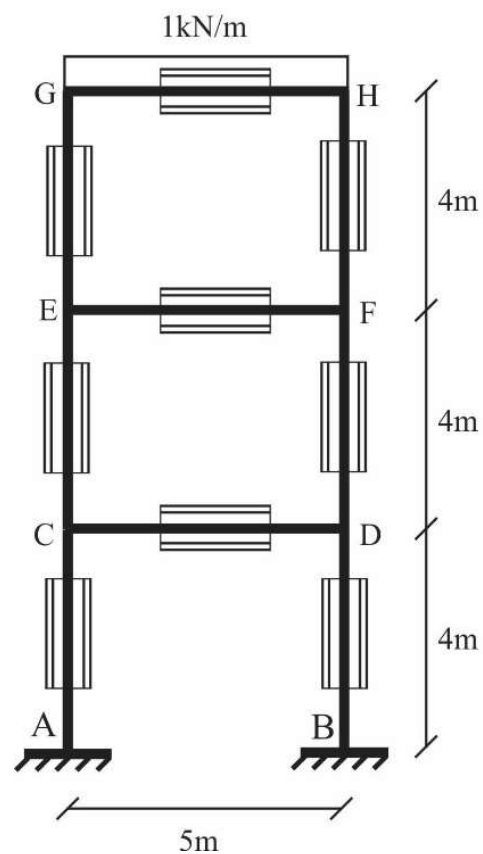

(a)

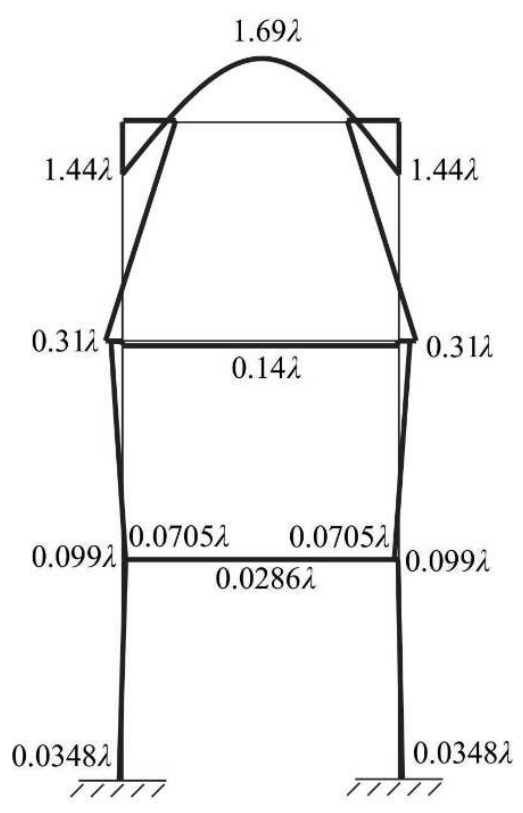

(b)

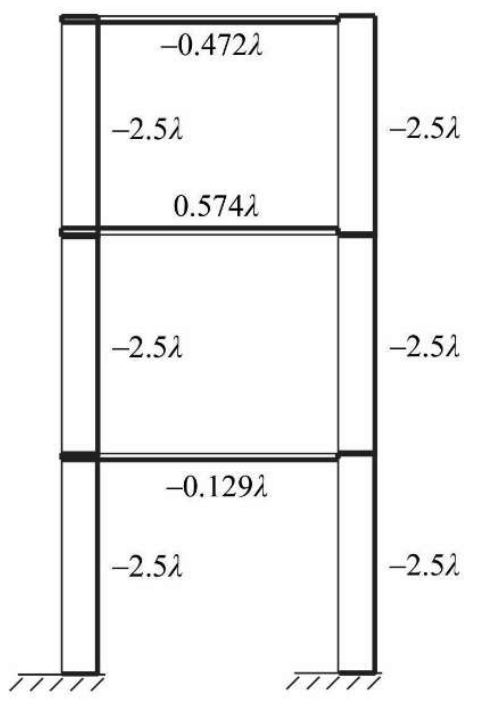

(c)

Fig. 11 Three -story frame under gravity load (a) Geometry, (b) Bending Moment diagram, and (c) Normal force diagram
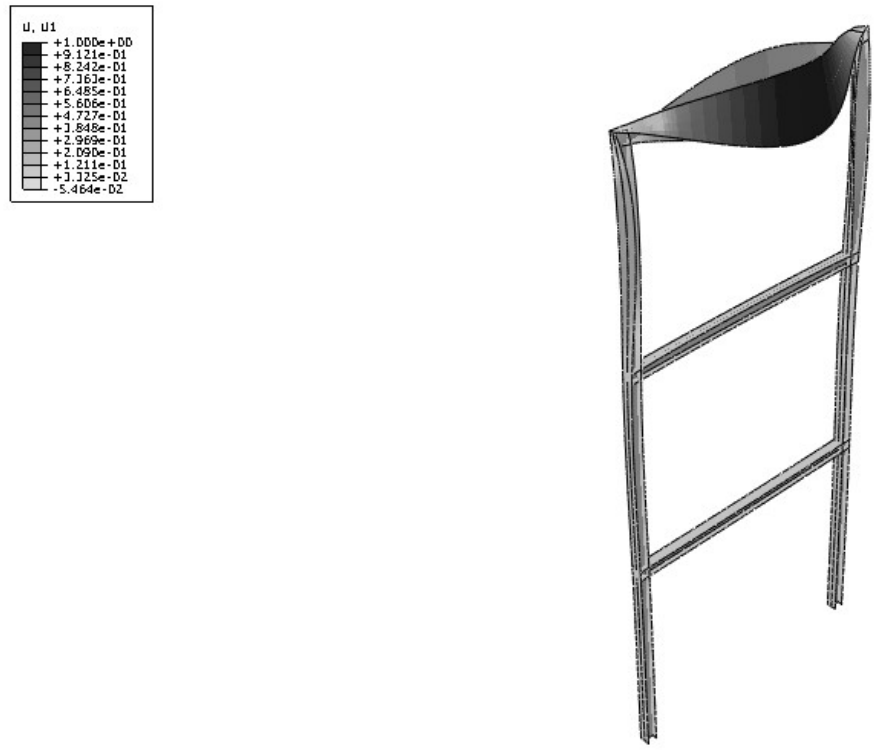

(a) 

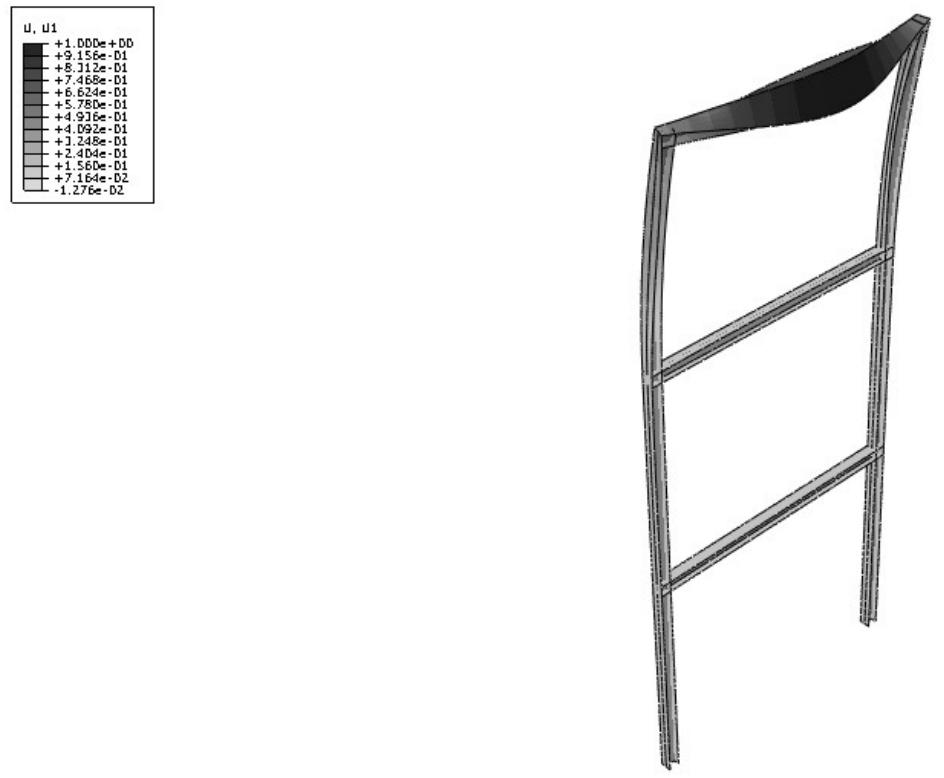

(b)

Fig. 12 buckling configurations for (a) Scenario (a) Frame laterally restrained at joints and (b) Scenario (b) Frame is laterally unrestrained at some of the joints

Table 4 Buckling load multipliers for 3-story frame with and without lateral restraints at junctions

\begin{tabular}{|c|c|c|c|c|c|c|c|}
\hline & & \multicolumn{3}{|c|}{ Scenario (1) with lateral restraints } \\
at joints & \multicolumn{3}{|c|}{$\begin{array}{c}\text { Scenario (2) no lateral restraints } \\
\text { except at junctions E, F }\end{array}$} \\
\cline { 2 - 8 } Type of solution & $\begin{array}{c}\text { Number } \\
\text { of } \\
\text { degrees } \\
\text { of } \\
\text { freedom }\end{array}$ & $\begin{array}{c}\text { Load } \\
\text { multiplier } \\
\lambda\end{array}$ & $\begin{array}{c}\text { Critical } \\
\text { moment } \\
\text { kNm in } \\
\text { Member } \\
\text { BG }\end{array}$ & $\begin{array}{c}\text { Load } \\
\text { multiplier } \\
\text { relative to } \\
\lambda_{1}\end{array}$ & $\begin{array}{c}\text { Load } \\
\text { multiplier } \\
\lambda\end{array}$ & $\begin{array}{c}\text { Critical } \\
\text { moment } \\
\text { kNm in } \\
\text { Member } \\
\text { BG }\end{array}$ & $\begin{array}{c}\text { Load } \\
\text { multiplier } \\
\text { relative to } \\
\lambda_{1}\end{array}$ \\
\hline $\begin{array}{c}\lambda_{1}: \text { ABAQUS shell } \\
\text { model of the whole } \\
\text { structure (S4R } \\
\text { element) }\end{array}$ & 63,498 & 183.5 & 310.1 & ---- & 69.50 & 117.5 & - --- \\
\hline $\begin{array}{c}\lambda_{2}: \text { present Joint } \\
\text { element and shear } \\
\text { deformable } \\
\text { elements for the } \\
\text { whole structure }\end{array}$ & 567 & 186.7 & 315.3 & 1.02 & 74.11 & 125.2 & 1.07 \\
\hline $\begin{array}{c}\lambda_{3}: \text { Continuous } \\
\text { warping restraint } \\
\text { and ABAQUS } \\
\text { B31OS elements of } \\
\text { the whole structure }\end{array}$ & 497 & 207.0 & 349.6 & 1.13 & 77.66 & 131.2 & 1.12 \\
\hline
\end{tabular}




\subsection{Example 4: T-shaped frame}

The laterally unsupported T-shaped pipe support frame investigated by Mohareb and Dabbas [44] is revisited. Beam and column cross sections are W250x58 (Compact section for a yield strength of $350 \mathrm{MPa}$ ). The column is fixed at the base, and the frame is subjected to two point loads acting at the top flange of horizontal member (Fig.13). The joint is a typical moment connection with two pairs of stiffeners. The horizontal cantilever length, $L_{b}$, takes the values 1.0 and $2.0 \mathrm{~m}$ while the column height, $L_{c}$, takes the values $3.0,4.0,5.0$, and $6.0 \mathrm{~m}$, resulting in a total of eight combinations.

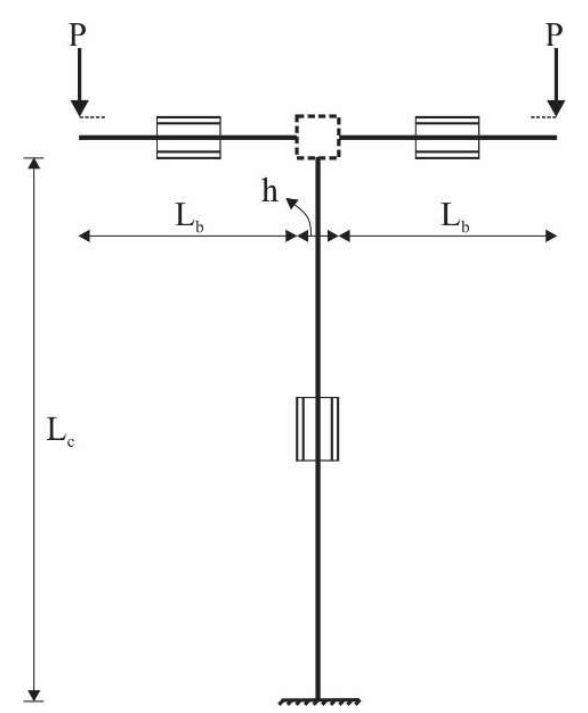

(a)

$\begin{array}{ll}\text { Width } b(\mathrm{~mm}) & 203.0 \\ \text { Height } h(\mathrm{~mm}) & 238.5 \\ \text { Flange thickness } t(\mathrm{~mm}) & 13.5 \\ \text { Web thickness } w(\mathrm{~mm}) & 8.0 \\ \text { Principal moment of inertia } I_{x}\left(\mathrm{~mm}^{4}\right) & 87.3 \times 10^{6} \\ \text { Principal moment of inertia } I_{y}\left(\mathrm{~mm}^{4}\right) & 18.8 \times 10^{6} \\ \text { Torsional constant } J\left(\mathrm{~mm}^{4}\right) & 409 \times 10^{3} \\ \text { Warping constant } I_{\omega}\left(\mathrm{mm}^{6}\right) & 2.68 \times 10^{11} \\ \text { Area } A\left(\mathrm{~mm}^{2}\right) & 7420\end{array}$

(b)

Fig. 13 T-shaped frame for Example 4 (a) Geometry and (b) Sectional properties for W250x58 section

The elastic critical load of the system is determined based on: (1) the present joint element formulation with shear deformable elements for other members of structure ( 8 elements per member, 24 in total) and (2) an ABAQUS S4R shell element as reported in Dabbas and Mohareb [44]. The cross-section was modeled using six elements along each flange, and eight elements along the web height. The number of elements $n_{L b}$ along the cantilever length and column height $n_{L c}$ are reported in Columns 3 and 4 of Table 5. The ratio of the critical loads predicted by the present solution to those predicted by ABAQUS ranges from 1.03 to 1.10, with an average of 1.07. 
As discussed in previous examples, the difference is attributed to distortional effects which are omitted in the present solution but captured in the shell finite element solution. Distortional effects tend to be minor for longer spans (e.g., $L_{c}=6 m$ and $L_{b}=2 m$ ) and gain significance for short spans (e.g., $L_{c}=3 m$ and $L_{b}=1 m$ ). In all cases, the present solution provides reasonably accurate buckling load predictions with a significantly smaller number of degrees of freedom.

Table 5 Buckling loads of a T-shaped structure with two pairs of stiffeners under two top flange loadings applying at tip

\begin{tabular}{|c|c|c|c|c|c|c|c|}
\hline \multicolumn{2}{|c|}{ Dimensions (m) } & \multicolumn{3}{|c|}{$\begin{array}{l}\text { Number of elements in Dabbas } \\
\text { and Mohareb [44] }\end{array}$} & \multicolumn{2}{|c|}{ Critical load (kN) } & \multirow[b]{2}{*}{$\begin{array}{c}\text { Ratio } \\
(8)=(6) /(7)\end{array}$} \\
\hline $\begin{array}{l}L_{c} \\
(1)\end{array}$ & $\begin{array}{l}L_{b} \\
(2)\end{array}$ & $\begin{array}{l}n_{L c} \\
(3)\end{array}$ & $\begin{array}{l}n_{L b} \\
(4)\end{array}$ & $\begin{array}{c}\text { Total } \\
(5)\end{array}$ & $\begin{array}{l}\text { Present study } \\
\text { (6) }\end{array}$ & $\begin{array}{c}\text { Dabbas and } \\
\text { Mohareb [44] } \\
\text { (7) }\end{array}$ & \\
\hline 3.0 & 1.0 & 93 & 31 & 3260 & 298 & 270 & 1.10 \\
\hline 4.0 & 1.0 & 124 & 31 & 3880 & 253 & 242 & 1.05 \\
\hline 5.0 & 1.0 & 155 & 31 & 4500 & 171 & 161 & 1.06 \\
\hline 6.0 & 1.0 & 186 & 31 & 5120 & 122 & 115 & 1.06 \\
\hline 3.0 & 2.0 & 93 & 62 & 4500 & 133 & 121 & 1.10 \\
\hline 4.0 & 2.0 & 124 & 62 & 5120 & 132 & 120 & 1.10 \\
\hline 5.0 & 2.0 & 155 & 62 & 5740 & 130 & 120 & 1.08 \\
\hline 6.0 & 2.0 & 186 & 62 & 6360 & 111 & 108 & 1.03 \\
\hline
\end{tabular}

\section{Summary and conclusions}

1. The present study developed a joint finite element that accurately quantifies the partial warping restraint provided by common moment connections to adjoining members framing at right angles.

2. The four-node joint element developed is based on an initial shell analysis for the joint and is condensed into a reduced 4 DOF element. The joint element interfaces seamlessly with the nodes of the classical 14 degrees of freedom beam finite element in [5] or that based on the shear deformable theory developed in [14].

3. The newly developed element provides a more accurate representation of the joint stiffness than the continuous warping deformation assumption and provides LTB moment estimates closer to those based on a shell-finite element analysis.

4. The developed joint element successfully extends functionality of existing LTB beam elements to analyze non-collinear structures and enables a more accurate and less un- 
conservative prediction of the elastic buckling resistance of plane frames when compared with a rigid joint representation. The gain in accuracy is achieved while keeping the degrees of freedom involved to a minimum.

5. The joint rotation effect is incorporated into the formulation. Its importance for the accurate prediction of frame buckling loads was illustrated through numerical examples.

6. Compared to the number of degrees of freedom involved in shell-finite element solutions, the newly developed joint element enables modelling the problem with fewer degrees of freedom. The underlying analysis can be conducted in a fraction of time and expense compared to shellbased solutions.

7. The limitations of the Salvadori hypothesis adopted in present design standards whereby the buckling loads for members are quantified by separating the members from the entire structure was illustrated through examples. The hypothesis is shown to lead to conservative results when frames are laterally restrained at the joints but can lead to un-conservative predictions when some of the frame joints are not laterally restrained. In contrast, the present solution accounts for interactions between members.

8. The joint element developed in the present study in conjunction with the shear deformable element in [14], provides a computationally effective means to predict the lateral buckling loads for multi-storey multi-bay plane frames where the use of shell models may be prohibitively computationally expensive.

\section{Acknowledgements}

The authors gratefully acknowledge financial support from the Natural Sciences and Engineering Research Council (NSERC) of Canada. 


\section{Appendix A - Lateral displacements due to warping}

This appendix provides the formulation on obtaining the lateral displacements induced by warping deformation. Four lateral displacement contributions result from each of warping deformations at each of the four faces of the joints. These contributions are provided in each of the following subsections.

\section{A.1. Contribution of warping at faces 1 and 2}

The displacements at Point 2 on face 2 are related to those of Point 1 at face 1 through point 5 located at their intersection (Fig. 6). The position vectors of points 1 and 2 relative to point 5 are:

$$
\begin{aligned}
& \left\langle x_{15}\right\rangle^{T}=\left\langle x_{1}\right\rangle^{T}-\left\langle x_{5}\right\rangle^{T}=\left\langle\begin{array}{lll}
0 & 0 & -h_{1} / 2
\end{array}\right\rangle \\
& \left\langle x_{25}\right\rangle^{T}=\left\langle x_{2}\right\rangle^{T}-\left\langle x_{5}\right\rangle^{T}=\left\langle\begin{array}{lll}
0 & -h_{2} / 2 & 0
\end{array}\right\rangle
\end{aligned}
$$

A positive warping deformation ${ }_{2}^{j k} \psi_{b}$ at face 2 induces a positive angle of rotation ${ }_{2}^{j k} \theta_{\psi b}=+\left(h_{2} / 2\right){ }_{2}^{j k} \psi_{b}$ about the $y_{j k}$ axis at Point 5 and an equal and opposite angle of rotation at point 6. Angle ${ }_{2}^{j k} \theta_{\psi b}$ at point 5 induces an additional lateral displacement at Point 1, $\left\langle{ }_{1}^{j k} U_{\psi^{2}}^{*}\right\rangle^{T}=\left\langle{ }_{1}^{j k} u_{b}^{*} \quad{ }_{1}^{j k} v_{p}^{*} \quad{ }_{1}^{j k} w_{p}^{*}\right\rangle^{T}$ given by

$\left\{{ }_{1}^{j k} U_{\psi 2}^{*}\right\}=\left[R_{\psi 2}\right]\left\{x_{15}\right\}$

Where the rotation matrix $\left[R_{\psi 2}\right]$ is defined as

$$
\left[R_{\psi 2}\right]_{3 \times 3}=\left[\begin{array}{ccc}
-\frac{1}{2}\left(\frac{h_{2}}{2}\right)^{2}\left({ }_{2}^{j k} \psi_{b}\right)^{2} & \frac{-h_{2}}{4} \lambda\left({ }_{2}^{j k} \theta_{x p}\right)\left({ }_{2}^{j k} \psi_{b}\right) & \frac{h_{2}}{2}\left({ }_{2}^{j k} \psi_{b}\right) \\
\frac{h_{2}}{4} \lambda\left({ }_{2}^{j k} \theta_{x p}\right)\left({ }_{2}^{j k} \psi_{b}\right) & -\frac{1}{2} \lambda^{2}\left({ }_{2}^{j k} \theta_{x p}\right)^{2} & -\lambda\left({ }_{2}^{j k} \theta_{x p}\right) \\
-\frac{h_{2}}{2}\left({ }_{2}^{j k} \psi_{b}\right) & \lambda\left({ }_{2}^{j k} \theta_{x p}\right) & -\frac{1}{2}\left[\lambda^{2}\left({ }_{2}^{j k} \theta_{x p}\right)^{2}+\left(\frac{h_{2}}{2}\right)^{2}\left({ }_{2}^{j k} \psi_{b}\right)^{2}\right.
\end{array}\right]
$$

The additional lateral displacement induced at Face 1 due to warping at face 2 is obtained by expanding the first row of right hand side of Eq. (A2) yielding

$$
{ }_{1}^{j k} u_{b}^{*}=-\left(h_{1} h_{2} / 4\right){ }_{2}^{j k} \psi_{b}
$$


Similarly, the additional displacement at Point $2,\left\langle{ }_{2}^{j k} U_{\psi 1}^{*}\right\rangle^{T}=\left\langle{ }_{2}^{j k} u_{b}^{*} \quad{ }_{2}^{j k} v_{p}^{*} \quad{ }_{2}^{j k} w_{p}^{*}\right\rangle^{T}$ is given by

$$
\left\{{ }_{2}^{j k} U_{\psi 1}^{*}\right\}=\left[R_{\psi 1}\right]\left\{x_{25}\right\}
$$

where

$$
\left[R_{\psi 1}\right]_{3 \times 3}=\left[\begin{array}{ccc}
-\frac{1}{2}\left(\frac{h_{1}}{2}\right)^{2}\left({ }_{1}^{j k} \psi_{b}\right)^{2} & -\frac{h_{1}}{2}\left({ }_{1}^{j k} \psi_{b}\right) & \frac{h_{1}}{4} \lambda\left({ }_{1}^{j k} \theta_{x p}\right)\left({ }_{1}^{j k} \psi_{b}\right) \\
\frac{h_{1}}{2}\left({ }_{1}^{j k} \psi_{b}\right) & -\frac{1}{2}\left(\frac{h_{1}}{2}\right)^{2}\left({ }_{1}^{j k} \psi_{b}\right)^{2} & -\lambda\left({ }_{1}^{j k} \theta_{x p}\right) \\
\frac{h_{1}}{4} \lambda\left({ }_{1}^{j k} \theta_{x p}\right)\left({ }_{1}^{j k} \psi_{b}\right) & \lambda\left({ }_{1}^{j k} \theta_{x p}\right) & -\frac{1}{2} \lambda^{2}\left({ }_{1}^{j k} \theta_{x p}\right)^{2}
\end{array}\right]
$$

The additional lateral displacement induced at Face 2 due to warping deformation at Face 1 is obtained by expanding the first row of the right hand side of Eq. (A5) yielding

$$
{ }_{2}^{j k} u_{b}^{*}=\left(h_{1} h_{2} / 4\right){ }_{1}^{j k} \psi_{b}
$$

The total out of plane displacement at point 2 obtained by summation of Eqs. (14a), (A4) and (A7) yielding

$$
-{ }_{2}^{j k} u_{b}+{ }_{1}^{j k} u_{b}-\frac{h_{1} h_{2}}{4}\left({ }_{2}^{j k} \psi_{b}\right)+\frac{h_{1}}{2}\left({ }_{1}^{j k} \theta_{y b}\right)+\frac{h_{2}}{2}\left({ }_{1}^{j k} \theta_{z b}\right)+\frac{h_{1} h_{2}}{4}\left({ }_{1}^{j k} \psi_{b}\right) \approx 0
$$

\section{A.2. Contributions of warping at faces 1 and 3}

Using the same methodology described in the previous Section [Eqs. (A2) and (A5)], the DOFs of node 2 are related to those of node 3. Then, using Eq. (A8) to relate the DOFs of node 2 to those of node 1, one can obtain the relationship between the DOFs of nodes 3 and 1 by eliminating DOFs of node 2. Displacements of node 2 are related to those of node 3 to form the rigid body motion as follows

$$
\left\{u_{2}\right\}_{3 \times 1}=\left\{u_{3}\right\}_{3 \times 1}+[R]_{3 \times 3}\left\{x_{23}\right\}_{3 \times 1}
$$

Expanding the right hand side of Eq. (A9) about the first row leads to

$$
{ }_{2}^{j k} u_{b}^{*}={ }_{3}^{j k} u_{b}^{*}-\frac{h_{2}}{2}\left({ }_{3}^{j k} \theta_{z b}\right)+\frac{h_{1}}{2}\left({ }_{3}^{j k} \theta_{y b}\right)
$$




\section{A.3. Contributions of warping at faces 2 and 3}

Displacements at point 2 of face 2 are related to those of at point 3 of face 3 through point 6 located at their junctions as follows

$$
\begin{aligned}
& \left\langle x_{36}\right\rangle^{T}=\left\langle x_{3}\right\rangle^{T}-\left\langle x_{6}\right\rangle^{T}=\left\langle\begin{array}{lll}
0 & 0 & -h_{1} / 2
\end{array}\right\rangle \\
& \left\langle x_{26}\right\rangle^{T}=\left\langle x_{2}\right\rangle^{T}-\left\langle x_{6}\right\rangle^{T}=\left\langle\begin{array}{lll}
0 & h_{2} / 2 & 0
\end{array}\right\rangle
\end{aligned}
$$

The angle ${ }_{2}^{j k} \theta_{\psi b}$ acting at point 6 induces an additional lateral displacement at point 3 , $\left\langle{ }_{3}^{j k} U_{\psi 2}^{*}\right\rangle^{T}=\left\langle{ }_{3}^{j k} u_{b}^{*} \quad{ }_{3}^{j k} v_{p}^{*} \quad{ }_{3}^{j k} w_{p}^{*}\right\rangle^{T}$ given by

$\left\{{ }_{3}^{j k} U_{\psi 2}^{*}\right\}=\left[R_{\psi 2}\right]\left\{x_{36}\right\}$

The additional lateral displacement induced at the face 3 due to warping deformation at face 2 is obtained by expanding the right hand side of Eq. (A12) about the first row as follows

$$
{ }_{3}^{j k} u_{b}^{*}=-\left(h_{1} h_{2} / 4\right){ }_{2}^{j k} \psi_{b}
$$

Similarly, the additional lateral displacement induced at the face 2 due to warping deformation at face 3 is obtained through

$\left\{{ }_{2}^{j k} U_{\psi 3}^{*}\right\}=\left[R_{\psi 3}\right]\left\{x_{26}\right\}$

Expanding the right hand side of Eq. (A14) about the first row leads to

$$
{ }_{2}^{j k} u_{b}^{*}=-\left(h_{1} h_{2} / 4\right){ }_{3}^{j k} \psi_{b}
$$

where

$$
\left[R_{\psi 3}\right]_{3 \times 3}=\left[\begin{array}{ccc}
-\frac{1}{2}\left(\frac{h_{1}}{2}\right)^{2}\left({ }_{3}^{j k} \psi_{b}\right)^{2} & -\frac{h_{1}}{2}\left({ }_{3}^{j k} \psi_{b}\right) & \frac{h_{1}}{4} \lambda\left({ }_{3}^{j k} \theta_{x p}\right)\left({ }_{3}^{j k} \psi_{b}\right) \\
\frac{h_{1}}{2}\left({ }_{3}^{j k} \psi_{b}\right) & -\frac{1}{2}\left(\frac{h_{1}}{2}\right)^{2}\left({ }_{3}^{j k} \psi_{b}\right)^{2} & -\lambda\left({ }_{3}^{j k} \theta_{x p}\right) \\
-\frac{h_{1}}{4} \lambda\left({ }_{3}^{j k} \theta_{x p}\right)\left({ }_{3}^{j k} \psi_{b}\right) & \lambda\left({ }_{3}^{j k} \theta_{x p}\right) & -\frac{1}{2} \lambda^{2}\left({ }_{3}^{j k} \theta_{x p}\right)^{2}
\end{array}\right]
$$

Finally, the total out of plane displacement at point 2 obtained by summation of Eqs. (A9), (A13) and (A15) resulting in

$$
-{ }_{2}^{j k} u_{b}+{ }_{3}^{j k} u_{b}-\frac{h_{2}}{2}\left({ }_{3}^{j k} \theta_{z b}\right)+\frac{h_{1}}{2}\left({ }_{3}^{j k} \theta_{y b}\right)-\left(\frac{h_{1} h_{2}}{4}\right){ }_{2}^{j k} \psi_{b}-\left(\frac{h_{1} h_{2}}{4}\right){ }_{3}^{j k} \psi_{b} \approx 0
$$


In Eq. (A8), DOFs of node 2 are related to those of node 1 through

$$
-{ }_{2}^{j k} u_{b}-\frac{h_{1} h_{2}}{4}\left({ }_{2}^{j k} \psi_{b}\right)+{ }_{1}^{j k} u_{b}+\frac{h_{1}}{2}\left({ }_{1}^{j k} \theta_{y b}\right)+\frac{h_{2}}{2}\left({ }_{1}^{j k} \theta_{z b}\right)+\frac{h_{1} h_{2}}{4}\left({ }_{1}^{j k} \psi_{b}\right) \approx 0
$$

Substituting Eq. (A17) into Eq. (A8) and using Eqs. (A8) and (10), $\left({ }_{3}^{j k} \theta_{y b}={ }_{1}^{j k} \theta_{y b},{ }_{3}^{j k} \theta_{z b}={ }_{1}^{j k} \theta_{z b}\right)$, DOFs of node 3 will be related to those of node 1 through

$-{ }_{3}^{j k} u_{b}+{ }_{1}^{j k} u_{b}+h_{2}\left({ }_{1}^{j k} \theta_{z b}\right)+\frac{h_{1} h_{2}}{4}\left({ }_{1}^{j k} \psi_{b}\right)+\frac{h_{1} h_{2}}{4}\left({ }_{3}^{j k} \psi_{b}\right) \approx 0$

\section{A.4. Contributions of warping at faces 1 and 4}

Displacements at point 4 of face 4 are related to those of at point 1 of face 1 through point 8 located at their junctions as follows

$$
\begin{aligned}
& \left\langle x_{18}\right\rangle^{T}=\left\langle x_{1}\right\rangle^{T}-\left\langle x_{8}\right\rangle^{T}=\left\langle\begin{array}{lll}
0 & 0 & h_{1} / 2
\end{array}\right. \\
& \left\langle x_{48}\right\rangle^{T}=\left\langle x_{4}\right\rangle^{T}-\left\langle x_{8}\right\rangle^{T}=\left\langle\begin{array}{lll}
0 & -h_{2} / 2 & 0
\end{array}\right.
\end{aligned}
$$

The angle ${ }_{4}^{j k} \theta_{\psi 4}$ acting at point 8 induces an additional lateral displacement at point 1 , $\left\langle{ }_{1}^{j k} U_{\psi 1}^{*}\right\rangle^{T}=\left\langle{ }_{1}^{j k} u_{b}^{*} \quad{ }_{1}^{j k} v_{p}^{*} \quad{ }_{1}^{j k} w_{p}^{*}\right\rangle^{T}$ given by

$\left\{{ }_{1}^{j k} U_{\psi 4}^{*}\right\}=\left[R_{\psi 4}\right]\left\{x_{18}\right\}$

The additional lateral displacement induced at the face 1 due to warping deformation at face 4 is obtained by expanding the right hand side of Eq. (A20) about the first row as follows

$$
{ }_{1}^{j k} u_{b}^{*}=\left(h_{1} h_{2} / 4\right){ }_{4}^{j k} \psi_{b}
$$

where

$$
\left.\left[R_{\psi 4}\right]_{3 \times 3}=\left[\begin{array}{ccc}
-\frac{1}{2}\left(\frac{h_{2}}{2}\right)^{2}\left({ }_{4}^{j k} \psi_{b}\right)^{2} & -\frac{h_{2}}{4} \lambda\left({ }_{4}^{j k} \theta_{x p}\right)\left({ }_{4}^{j k} \psi_{b}\right) & \frac{h_{2}}{2}\left({ }_{4}^{j k} \psi_{b}\right) \\
\frac{h_{2}}{4} \lambda\left({ }_{4}^{j k} \theta_{x p}\right)\left({ }_{4}^{j k} \psi_{b}\right) & -\frac{1}{2} \lambda^{2}\left({ }_{4}^{j k} \theta_{x p}\right)^{2} & -\lambda\left({ }_{4}^{j k} \theta_{x p}\right) \\
-\frac{h_{2}}{2}\left({ }_{4}^{j k} \psi_{b}\right) & \lambda\left({ }_{4}^{j k} \theta_{x p}\right) & -\frac{1}{2}\left[\lambda^{2}\left({ }_{4}^{j k} \theta_{x p}\right)^{2}+\left(\frac{h_{2}}{2}\right)^{2}\left({ }_{4}^{j k} \psi_{b}\right)^{2}\right.
\end{array}\right]\right]
$$

Similarly, on has

$$
\left\{{ }_{4}^{j k} U_{\psi 1}^{*}\right\}=\left[R_{\psi 1}\right]\left\{x_{48}\right\}
$$


Similarly, the additional lateral displacement induced at the face 4 due to warping deformation at face 1 is obtained by expanding the right hand side of Eq. (A23) about the first row as follows ${ }_{4}^{j k} u_{b}^{*}=\left(h_{1} h_{2} / 4\right){ }_{1}^{j k} \psi_{b}$

Finally, the total out of plane displacement at point 4 is obtained by summation of Eqs. (14c), (A21) and (A24)

$-{ }_{4}^{j k} u_{b}+{ }_{1}^{j k} u_{b}+\frac{h_{1} h_{2}}{4}\left({ }_{4}^{j k} \psi_{b}\right)-\frac{h_{1}}{2}\left({ }_{1}^{j k} \theta_{y b}\right)+\frac{h_{2}}{2}\left({ }_{1}^{j k} \theta_{z b}\right)+\frac{h_{1} h_{2}}{4}\left({ }_{1}^{j k} \psi_{b}\right) \approx 0$ 


\section{Appendix B - Bimoments due to warping deformation}

This appendix presents the formulation on obtaining the bimoments induced by warping deformation on Faces 2 to 4. Using the same methodology depicted in Section 5.3, bimoments induced on Face 2 can be determined through the following Eq. (B1a-d) as shown in Fig. B1.

$$
\begin{aligned}
& { }_{12}^{j k} B_{b l}=\int_{A_{1}} \sigma_{1} \omega_{1}(s) d A \approx \sum_{r=1}^{n r}\left({ }^{j k} R_{y r 5^{\prime}-5^{n}}\right)\left(\frac{-h_{1}}{2} x_{r 5^{\prime}-5^{\prime \prime}}\right)+\sum_{r=1}^{n r}\left({ }^{j k} R_{y r 8^{\prime}-8^{\prime \prime}}\right)\left(\frac{h_{1}}{2} x_{r 8^{\prime}-8^{\prime \prime}}\right) \\
& { }_{22}^{j k} B_{b l}=\int_{A_{1}} \sigma_{2} \omega_{2}(s) d A \approx \sum_{r=1}^{n r}\left({ }^{j k} R_{z r 5^{\prime}-5^{\prime \prime}}\right)\left(\frac{-h_{2}}{2} x_{r 5^{\prime}-5^{\prime \prime}}\right)+\sum_{r=1}^{n r}\left({ }^{j k} R_{z r 6^{6}-6^{\prime \prime}}\right)\left(\frac{h_{2}}{2} x_{r 6^{\prime}-6^{\prime \prime}}\right) \\
& { }_{32}^{j k} B_{b l}=\int_{A_{1}} \sigma_{3} \omega_{3}(s) d A \approx \sum_{r=1}^{n r}\left(R_{y r 6^{\prime}-6^{\prime \prime}}\right)\left(\frac{h_{1}}{2} x_{r 6^{\prime}-6^{\prime \prime}}\right)+\sum_{r=1}^{n r}\left({ }^{j k} R_{y r 7^{\prime}-7^{\prime \prime}}\right)\left(\frac{-h_{1}}{2} x_{r 7^{\prime}-7^{\prime \prime}}\right) \\
& { }_{42}^{j k} B_{b l}=\int_{A_{1}} \sigma_{4} \omega_{4}(s) d A \approx \sum_{r=1}^{n r}\left({ }^{j k} R_{z r 8^{\prime}-8^{\prime \prime}}\right)\left(\frac{h_{2}}{2} x_{r 8^{\prime}-8^{\prime \prime}}\right)+\sum_{r=1}^{n r}\left({ }^{j k} R_{z r 7^{\prime}-7^{\prime \prime}}\right)\left(\frac{-h_{2}}{2} x_{r 7^{\prime}-7^{\prime \prime}}\right)
\end{aligned}
$$

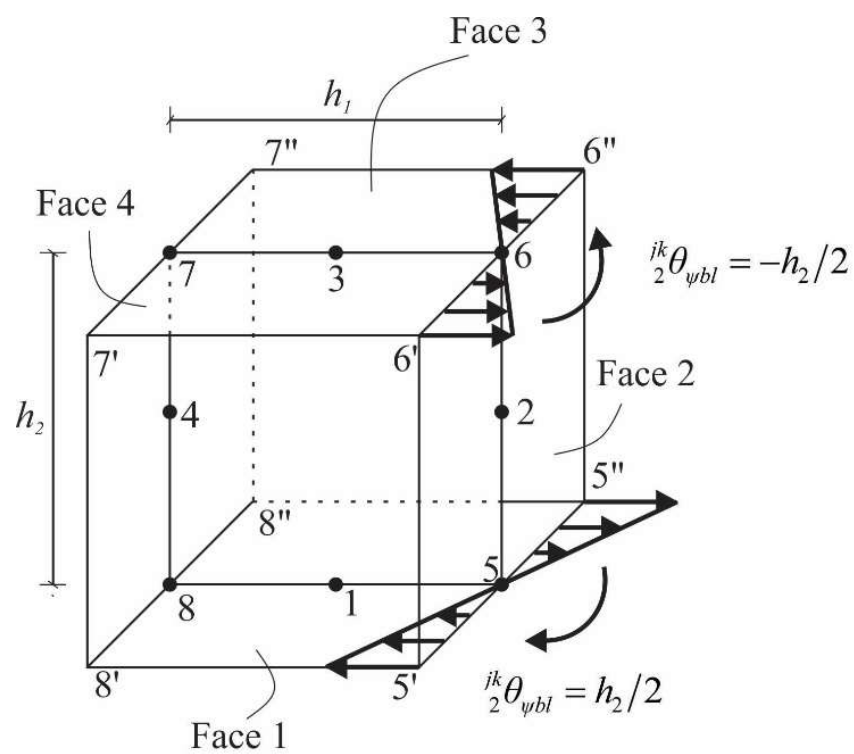

Face 1

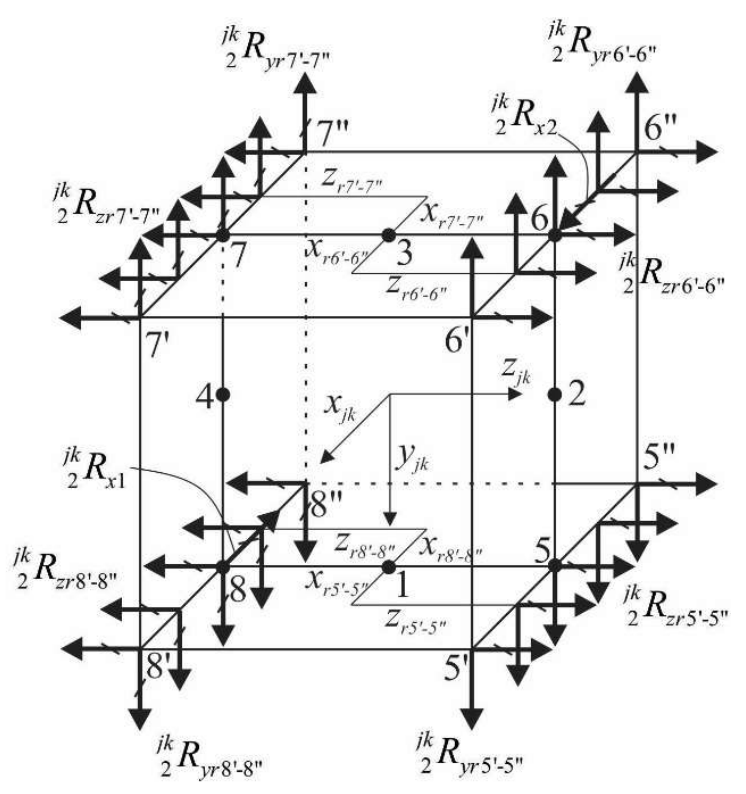

Fig. B1 (a) Unit warping deformation applied at Face 2; (b) boundary conditions and reactions

As shown in Fig. B2 and Fig. B3, are determined from 


$$
\begin{aligned}
& { }_{13}^{j k} B_{b l}=\int_{A_{1}} \sigma_{1} \omega_{1}(s) d A \approx \sum_{r=1}^{n r}\left({ }^{j k} R_{y r 5^{\prime}-5^{\prime \prime}}\right)\left(\frac{-h_{1}}{2} x_{r 5^{\prime}-5^{\prime \prime}}\right)+\sum_{r=1}^{n r}\left({ }^{j k} R_{y r 8^{\prime}-8^{\prime \prime}}\right)\left(\frac{h_{1}}{2} x_{r 8^{\prime}-8^{\prime \prime}}\right) \\
& { }_{23}^{j k} B_{b l}=\int_{A_{1}} \sigma_{2} \omega_{2}(s) d A \approx \sum_{r=1}^{n r}\left({ }^{j k} R_{z r 5^{\prime}-5^{\prime \prime}}\right)\left(\frac{-h_{2}}{2} x_{r 5^{\prime}-5^{\prime \prime}}\right)+\sum_{r=1}^{n r}\left({ }^{j k} R_{z r 6^{\prime}-6^{\prime \prime}}\right)\left(\frac{h_{2}}{2} x_{r 6^{\prime}-6^{\prime \prime}}\right) \\
& { }_{33}^{j k} B_{b l}=\int_{A_{1}} \sigma_{3} \omega_{3}(s) d A \approx \sum_{r=1}^{n r}\left(R_{y r 6^{\prime}-6^{\prime \prime}}\right)\left(\frac{h_{1}}{2} x_{r 6^{\prime}-6^{\prime \prime}}\right)+\sum_{r=1}^{n r}\left({ }^{j k} R_{y r 7^{\prime}-7^{\prime \prime}}\right)\left(\frac{-h_{1}}{2} x_{r 7^{\prime}-7^{\prime \prime}}\right) \\
& { }_{43}^{j k} B_{b l}=\int_{A_{1}} \sigma_{4} \omega_{4}(s) d A \approx \sum_{r=1}^{n r}\left({ }^{j k} R_{z r 8^{\prime}-8^{\prime \prime}}\right)\left(\frac{h_{2}}{2} x_{r 8^{\prime}-8^{\prime \prime}}\right)+\sum_{r=1}^{n r}\left({ }^{j k} R_{z r 7^{\prime}-7^{\prime \prime}}\right)\left(\frac{-h_{2}}{2} x_{r 7^{\prime}-7^{\prime \prime}}\right) \\
& { }_{14}^{j k} B_{b l}=\int_{A_{1}} \sigma_{1} \omega_{1}(s) d A \approx \sum_{r=1}^{n r}\left({ }^{j k} R_{y r 5^{\prime}-5^{\prime \prime}}\right)\left(\frac{-h_{1}}{2} x_{r 5^{\prime}-5^{\prime \prime}}\right)+\sum_{r=1}^{n r}\left({ }^{j k} R_{y r 8^{\prime}-8^{\prime \prime}}\right)\left(\frac{h_{1}}{2} x_{r 8^{\prime}-8^{\prime \prime}}\right) \\
& { }_{{ }_{34} k} B_{b l}=\int_{A_{1}} \sigma_{2} \omega_{2}(s) d A \approx \sum_{A_{1}}^{n r}\left({ }^{j k} R_{z r 5^{\prime}-5^{\prime \prime}}\right)\left(\frac{-h_{2}}{2} x_{r 5^{\prime}-5^{\prime \prime}}\right)+\sum_{r=1}^{n r}\left({ }^{j k} R_{z r 6^{\prime}-6^{\prime \prime}}\right)\left(\frac{h_{2}}{2} x_{r 6^{\prime}-6^{\prime \prime}}\right) d A \approx \sum_{r=1}^{n r}\left(R_{y r 6^{\prime}-6^{\prime \prime}}\right)\left(\frac{h_{1}}{2} x_{r 6^{\prime}-6^{\prime \prime}}\right)+\sum_{r=1}^{n r}\left({ }^{j k} R_{y r 7^{\prime}-7^{\prime \prime}}\right)\left(\frac{-h_{1}}{2} x_{r 7^{\prime}-7^{\prime \prime}}\right) \\
& { }_{44}^{j k} B_{b l}=\int_{A_{1}} \sigma_{4} \omega_{4}(s) d A \approx \sum_{r=1}^{n r}\left({ }^{j k} R_{z r 8^{\prime}-8^{\prime \prime}}\right)\left(\frac{h_{2}}{2} x_{r 8^{\prime}-8^{\prime \prime}}\right)+\sum_{r=1}^{n r}\left({ }^{j k} R_{z r 7^{\prime}-7^{\prime \prime}}\right)\left(\frac{-h_{2}}{2} x_{r 7^{\prime}-7^{\prime \prime}}\right)
\end{aligned}
$$

Face 3

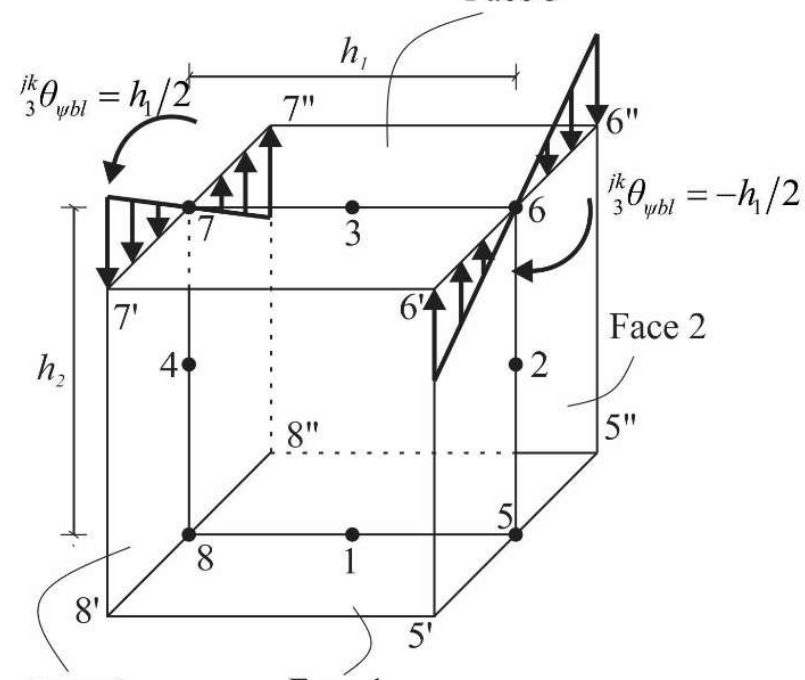

Face 4

Face 1

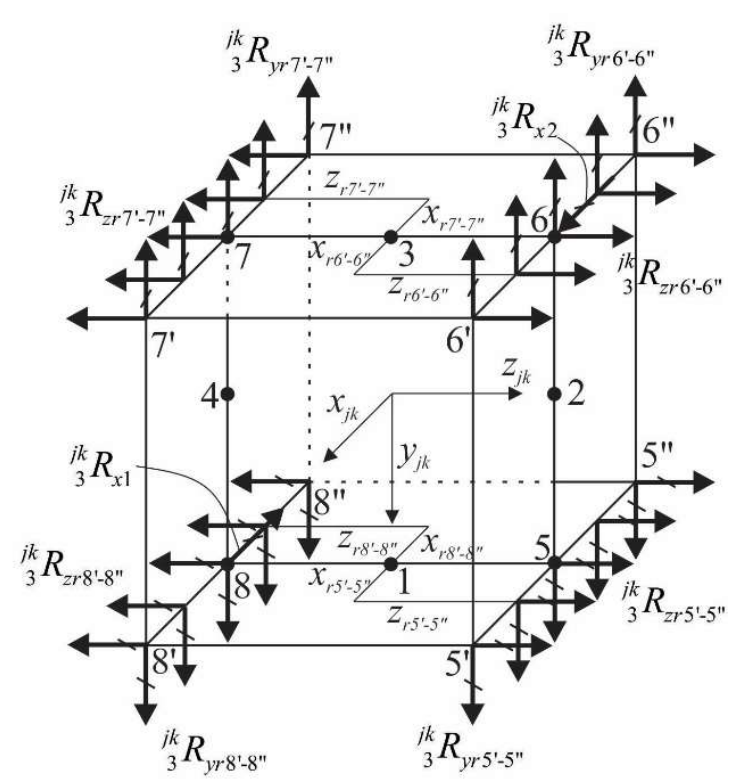

Fig. B2 (a) Unit warping deformation applied at Face 3; (b) boundary conditions and reactions 
Face 3

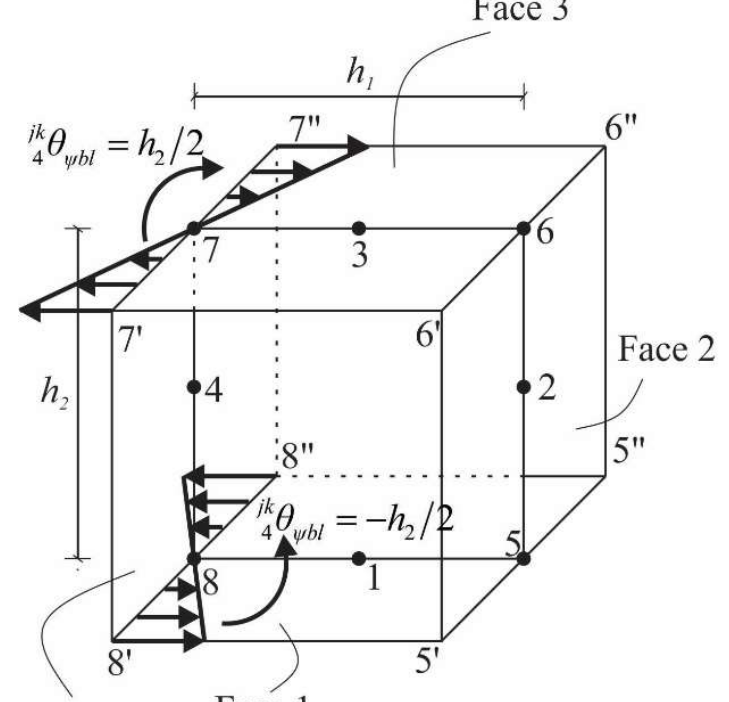

Face $4 \quad$ Face 1

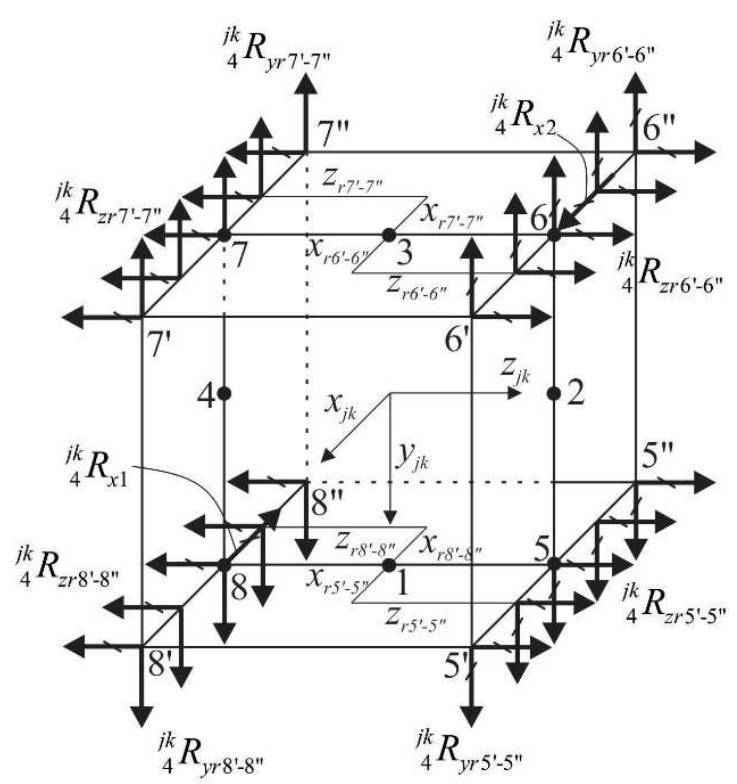

Fig. B3 (a) Unit warping deformation applied at Face 4; (b) boundary conditions and reactions 


\section{Appendix C - Entries of the warping spring stiffness matrices}

This section provides the entries of coupled spring warping stiffness matrices obtained from shell analysis to determine the critical moment for the Examples 1-4. All units are $\mathrm{Nmm}^{2} / \mathrm{rad}$ in all Cases. For the Examples 1 and 2, the section used is W200x59 and the coupled warping stiffness matrix takes the form

$$
\left[{ }^{j k} K_{\psi b l}\right]_{4 \times 4}=10^{13} \times\left[\begin{array}{cccc}
27.2 & -6.99 & 12.7 & -6.98 \\
-6.99 & 27.2 & 6.98 & -12.7 \\
12.7 & 6.98 & 27.2 & 6.99 \\
-6.98 & -12.7 & 6.99 & 27.2
\end{array}\right]
$$

Also, the $3 \times 3$ and $2 \times 2$ versions at the left and right of the frame are given by

$$
\left[{ }^{j k} K_{\psi b l}\right]_{3 \times 3}=10^{14} \times\left[\begin{array}{ccc}
2.54 & -1.02 & 1.45 \\
-1.02 & 2.13 & 1.02 \\
1.45 & 1.02 & 2.54
\end{array}\right], \quad\left[{ }^{j k} K_{\psi b l}\right]_{2 \times 2}=10^{14} \times\left[\begin{array}{cc}
1.72 & -1.61 \\
-1.61 & 1.72
\end{array}\right]
$$

For the Example 3, the cross-section is W200x46 and the corresponding warping stiffness matrix takes the form

$$
\left[{ }^{j k} K_{\psi b l}\right]_{4 \times 4}=10^{13} \times\left[\begin{array}{cccc}
20.6 & -5.26 & 9.83 & -5.26 \\
-5.26 & 20.6 & 5.26 & -9.83 \\
9.83 & 5.26 & 20.6 & 5.26 \\
-5.26 & -9.83 & 5.26 & 20.6
\end{array}\right]
$$

Again, the $3 \times 3$ and $2 \times 2$ versions at the left and right of the frame are given by

$$
\left[{ }^{j k} K_{\psi b l}\right]_{3 \times 3}=10^{13} \times\left[\begin{array}{ccc}
19.3 & -7.77 & 11.2 \\
-7.77 & 15.9 & 7.77 \\
11.2 & 7.77 & 19.3
\end{array}\right], \quad\left[{ }^{j k} K_{\psi b l}\right]_{2 \times 2}=10^{14} \times\left[\begin{array}{cc}
1.28 & -1.23 \\
-1.23 & 1.28
\end{array}\right]
$$

For the Example 4, the cross-section is W250x58 and the corresponding warping stiffness matrix takes the form 
$\left[{ }^{j k} K_{\psi b l}\right]_{4 \times 4}=10^{14} \times\left[\begin{array}{cccc}3.36 & -1.07 & 1.17 & -1.07 \\ -1.07 & 3.36 & 1.07 & -1.17 \\ 1.17 & 1.07 & 3.36 & 1.07 \\ -1.07 & -1.17 & 1.07 & 3.36\end{array}\right]$

and the $3 \times 3$ version depicted as configuration 5 in Table 1 is given by

$$
\left[{ }^{j k} K_{\psi b l}\right]_{3 \times 3}=10^{14} \times\left[\begin{array}{ccc}
2.96 & -1.44 & -1.44 \\
-1.44 & 3.02 & -1.51 \\
-1.44 & -1.51 & 3.02
\end{array}\right]
$$




\section{List of Symbols}

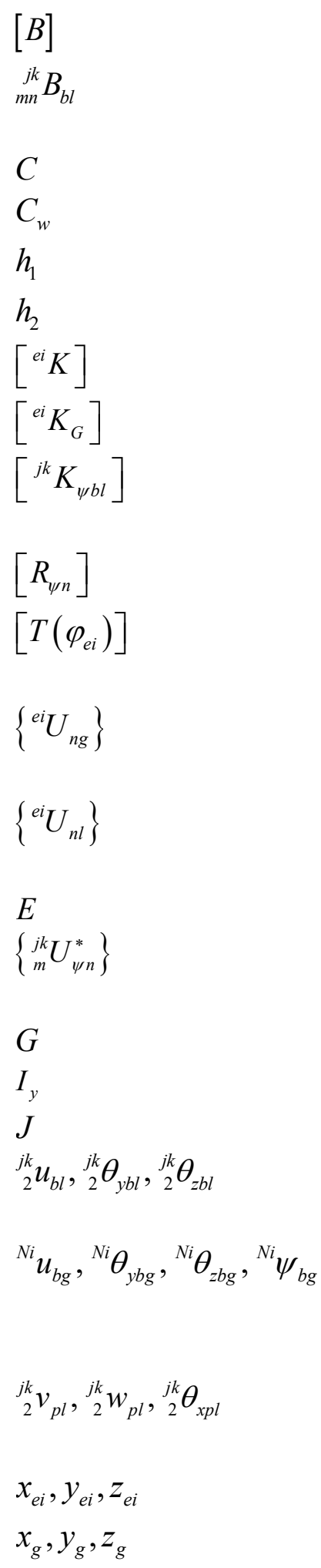

A user-input coefficient matrix

Bimoment at face $m$ induced by warping at face $n$ in local coordinates of joint $k$

Moment gradient factor

Warping Constant

Width of Face 1

Height of Face 2

Elastic stiffness matrix of member element $i$

Geometric stiffness matrix of member element $i$

Buckling warping matrix relates the bimoments to warping deformations

Rotation matrix induced by warping on face $n$, that is, $\left({ }_{n}^{j k} \psi_{b l}\right)$

Transformation matrix of member element $i$ from global coordinates to local coordinates

Vector of buckling nodal displacements of member element $i$ in global coordinates

Vector of buckling nodal displacements of member element $i$ in local coordinates

Modulus of elasticity

Additional displacement vector at point $m$ because of warping at face $n$ of joint $k$

Shear modulus

Moment of inertia of the cross-section about y-axis

St. Venant torsional constant

Buckling displacement and rotations of the second node in the local coordinates of joint element $k$

Buckling displacement and rotations of virtual node $i$ in global coordinates, and the node connects a member element and a joint: $i=1,2,3,4$

Pre-buckling displacement and rotations of the second node in the local coordinates of joint element $k$

Local coordinates for member element $i$

Global coordinates for the frame 


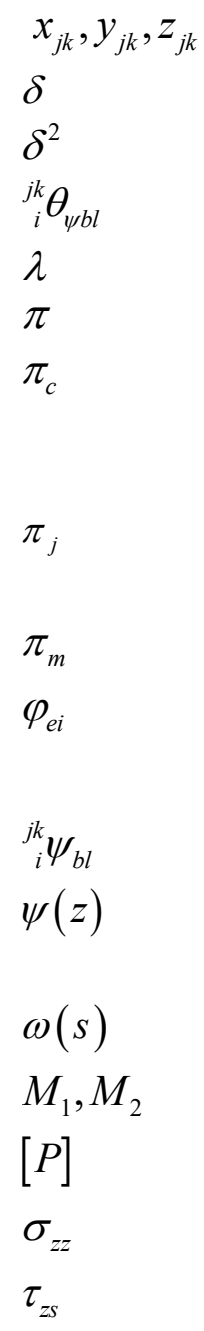

Local coordinates for joint element $k$

First variation of the argument functional

Second variation of the argument functional

Rotation angle of the edges at Face $i$ as a result of warping

Load factor (eigenvalue)

Total potential energy of a frame

Additional energy term enforcing rigid body constraints between the

degrees of freedom of Nodes 1, 2, 3 and 4 of joint elements through

Lagrange's multipliers

Total potential energy of all the joint elements because of buckling warping deformation

Total potential energy for all collinear member elements

Orientation of member element $i$, which is positively defined when

global axis $z_{g}$ rotates counter clockwise to local axis $z_{e i}$

Warping of the joint element $k$ at Face $i$

Rate of change of the longitudinal displacement as a result of warping along the z-axis (1/length dimension); and

Warping function (sectorial area) of a point $s$

Internal bending moment at both end of an element

Permutation matrix

Normal stress along $\mathrm{z}$ direction

Shear stress on the cross-section mid-surface

\section{References}

[1] Canadian Standards Association, (CSA), Limit States Design of Steel Structures, CAN/CSA S16-14, in, Toronto, Ontario, Canada, 2014.

[2] American Institute of Steel Construction, (AISC), Specification for Structural Steel Buildings, ANSI/AISC 360-16, AISC, Chicago, IL, 2016.

[3] M.G. Salvadori, Stability of I-Beams under thrust and unequal end moments, Proceeding ASCE, 79(291) (1953) 1-25.

[4] D. Krajcinovic, A consistent discrete elements technique for thinwalled assemblages, Int. J. of Solids and Struct., 5 (1969) 639-662.

[5] R.S. Barsoum, R.H. Gallagher, Finite element analysis of torsional and torsional-flexural stability problems, International Journal for Numerical Methods in Engineering, 2 (1970) 335-352. [6] G. Powell, R. Klingner, Elastic Lateral Buckling of Steel Beams, J. of the Struct. Div., 96 (1970) 1919-1932. 
[7] R.E. Erkmen, M. Mohareb, Buckling analysis of thin-walled open members - A finite element formulation, Thin Wall Struct., 46 (2008b) 618-636.

[8] R.E. Erkmen, M. Mohareb, Buckling analysis of thin-walled open members-A complementary energy variational principle, Thin Wall Struct., 46 (2008a) 602-617.

[9] L. Wu, M. Mohareb, Buckling of shear deformable thin-walled members-I. Variational principle and analytical solutions, Thin Wall Struct., 49 (2011a) 197-207.

[10] L. Wu, M. Mohareb, Buckling formulation for shear deformable thin-walled members-II. Finite element formulation, Thin Wall Struct., 49 (2011b) 208-222.

[11] M.M. Attard, M.-Y. Kim, Lateral buckling of beams with shear deformations - A hyperelastic formulation, Int. J. of Solids and Struct., 47 (2010) 2825-2840.

[12] R.E. Erkmen, M.M. Attard, Lateral-torsional buckling analysis of thin-walled beams including shear and pre-buckling deformation effects, Int. J. of Mech. Sci., 53 (2011) 918-925.

[13] R.E. Erkmen, Shear deformable hybrid finite-element formulation for buckling analysis of thin-walled members, Finite Elements in Analysis and Design, 82 (2014) 32-45.

[14] A. Sahraei, M. Mohareb, Upper and lower bound solutions for lateral-torsional buckling of doubly symmetric members, Thin Wall Struct., 102 (2016) 180-196.

[15] S. Kitipornchai, C. Wang, N. Trahair, Buckling of Monosymmetric I-Beams under Moment Gradient, J. of Struct. Eng., 112 (1986) 781-799.

[16] C. Wang, S. Kitipornchai, Buckling Capacities of Monosymmetric I-Beams, J. of Struct. Eng., 112 (1986) 2373-2391.

[17] F. Mohri, A. Brouki, J.C. Roth, Theoretical and numerical stability analyses of unrestrained, mono-symmetric thin-walled beams, J. of Constr. Steel Res., 59 (2003) 63-90.

[18] F. Mohri, N. Damil, M. Potier-Ferry, Linear and non-linear stability analyses of thin-walled beams with monosymmetric I sections, Thin Wall Struct., 48 (2010) 299-315.

[19] F. Mohri, N. Damil, M. Potier-Ferry, Buckling and lateral buckling interaction in thin-walled beam-column elements with mono-symmetric cross sections, Appl. Math. Mod., 37 (2013) 35263540 .

[20] A. Andrade, D. Camotim, P. Providência e Costa, On the evaluation of elastic critical moments in doubly and singly symmetric I-section cantilevers, J. of Constr. Steel Res., 63 (2007) 894-908.

[21] L. Zhang, G.S. Tong, Elastic flexural-torsional buckling of thin-walled cantilevers, Thin Wall Struct., 46 (2008) 27-37.

[22] A. Sahraei, L. Wu, M. Mohareb, Finite element formulation for lateral torsional buckling analysis of shear deformable mono-symmetric thin-walled members, Thin Wall Struct., 89 (2015) 212-226.

[23] C.P. Johnson, K.M. Will, Beam buckling by finite element procedure, J. of the Struct. Div., $100(3)$ (1974) 669-685.

[24] G.J. Hancock, M.A. Bradford, N.S. Trahair, Web distortion and flexural-torsional buckling, J. of the Struct. Div., 106(7) (1980) 1557-1571.

[25] M.A. Bradford, N.S. Trahair, Distortional buckling of thin-web beam-columns, Eng. Struct., 4 (1982) 2-10.

[26] M.A. Bradford, N.S. Trahair, Distortional buckling of I-beams, J. of the Struct. Div., 107(2) (1981) 355-370.

[27] M.A. Bradford, Distortional buckling of monosymmetric I-beams, J. of Constr. Steel Res., 5 (1985) 123-136. 
[28] M.A. Bradford, Inelastic distortional buckling of I-beams, Comput. \& Struct., 24 (1986) 923933.

[29] M.A. Bradford, Buckling of doubly-symmetric cantilevers with slender webs, Eng. Struct., 14 (1992a) 327-334.

[30] M.A. Bradford, Lateral-Distortional buckling of steel I-Section members, J. of Constr. Steel Res., 23 (1992b) 97-116.

[31] M. Bradford, H. Ronagh, Generalized Elastic Buckling of Restrained I-Beams by FEM, J. of Struct. Eng., 123 (1997) 1631-1637.

[32] R. Hassan, M. Mohareb, Distortional lateral torsional buckling for simply supported beams with web cleats, Can. J. of Civil Eng., 42 (2015) 1091-1103.

[33] P. Pezeshky, A. Sahraei, M. Mohareb, Distortional Lateral Torsional Buckling Analysis of Beams with Overhangs, Journal of Structural Engineering, ASCE, in press (2018).

[34] P. Pezeshky, M. Mohareb, Distortional lateral torsional buckling of beam-columns including pre-buckling deformation effects, Comput. \& Struct., (2018).

[35] A.J. Hartmann, W.H. Munse, Flexural torsional buckling of planar frames, Journal of Engineering Mechanics Division, 92 (1966) 37-60.

[36] P. Vacharajittiphan, N.S. Trahair, Warping and distortion at I-section Joints, J. of the Struct. Div., 100 (1974) 547-564.

[37] P.J.B. Morrel, The influence of joint detail on the torsional behaviour of thin-walled structures having an axial discontinuity, in: Proceeding International Conference Thin-Walled Structures, University of Strathclyde, Glasgow, Scotland, 1979, pp. 539-556.

[38] M.M. Ettouney, J.B. Kirby, Warping restraint in threedimensional frames, J. of the Struct. Div., 107 (1981) 1643-1656.

[39] Y.-B. Yang, W. McGuire, A procedure for analysing space frames with partial warping restraint, International Journal for Numerical Methods in Engineering, 20 (1984) 1377-1398.

[40] P.W. Sharman, Analysis of structures with thin-walled open sections, Int. J. of Mech. Sci., 27 (1985) 665-677.

[41] S. Krenk, L. Damkilde, Warping of Joints in I-Beam Assemblages, J. of Eng. Mech., 117 (1991) 2457-2474.

[42] Y. Pi, N. Trahair, Distortion and Warping at Beam Supports, J. of Struct. Eng., 126 (2000) $1279-1287$.

[43] A. Masarira, The effect of joints on the stability behaviour of steel frame beams, J. of Constr. Steel Res., 58 (2002) 1375-1390.

[44] M. Mohareb, A. Dabbas, Lateral stability of partially restrained cantilever supports, in: Proceedings of the Annual Stability Conference, Baltimore, Maryland, 2003, pp. 2-5.

[45] I. Zinoviev, M. Mohareb, Analysis and design of laterally unsupported portal frames for outof-plane stability, Can. J. of Civil Eng., 31 (2004) 440-452.

[46] G.S. Tong, X.X. Yan, L. Zhang, Warping and bimoment transmission through diagonally stiffened beam-to-column joints, J. of Constr. Steel Res., 61 (2005) 749-763.

[47] C. Basaglia, D. Camotim, N. Silvestre, Torsion warping transmission at thin-walled frame joints: Kinematics, modelling and structural response, J. of Constr. Steel Res., 69 (2012) 39-53.

[48] S. Shayan, K.J.R. Rasmussen, A model for warping transmission through joints of steel frames, Thin Wall Struct., 82 (2014) 1-12.

[49] L. Wu, M. Mohareb, Finite-Element Formulation for the Lateral Torsional Buckling of Plane Frames, J. of Eng. Mech., 139 (2012) 512-524. 
[50] Z.P. Bazant, L. Cedolin, Stability of structures, Oxford University Press, Oxford, U.K., 1991. [51] N.L. Rizzi, V. Varano, The effects of warping on the postbuckling behaviour of thin-walled structures, Thin Wall Struct., 49 (2011) 1091-1097.

[52] N.S. Trahair, Flexural-Torsional Buckling of Structures, CRC Press, USA, 1993. 